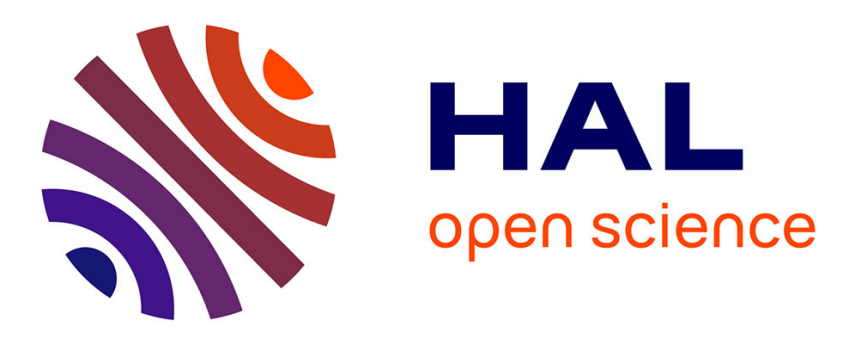

\title{
Chromosomes function as a barrier to mitotic spindle bipolarity in polyploid cells
}

\author{
Alix Goupil, Maddalena Nano, Gaëlle Letort, Simon Gemble, Frances \\ Edwards, Oumou Goundiam, Delphine Gogendeau, Carole Pennetier, Renata \\ Basto
}

\section{To cite this version:}

Alix Goupil, Maddalena Nano, Gaëlle Letort, Simon Gemble, Frances Edwards, et al.. Chromosomes function as a barrier to mitotic spindle bipolarity in polyploid cells. Journal of Cell Biology, 2020, 219 (4), pp.e201908006. 10.1083/jcb.201908006 . hal-03454921

\section{HAL Id: hal-03454921 \\ https://hal.science/hal-03454921}

Submitted on 29 Nov 2021

HAL is a multi-disciplinary open access archive for the deposit and dissemination of scientific research documents, whether they are published or not. The documents may come from teaching and research institutions in France or abroad, or from public or private research centers.
L'archive ouverte pluridisciplinaire HAL, est destinée au dépôt et à la diffusion de documents scientifiques de niveau recherche, publiés ou non, émanant des établissements d'enseignement et de recherche français ou étrangers, des laboratoires publics ou privés. 


\title{
ARTICLE
}

\section{Chromosomes function as a barrier to mitotic spindle bipolarity in polyploid cells}

\author{
Alix Goupil ${ }^{* 1}$, Maddalena Nano*1 (D), Gaëlle Letort ${ }^{2}$ (D), Simon Gemble ${ }^{1}$ (D), Frances Edwards ${ }^{1}$, Oumou Goundiam ${ }^{1,3}$, Delphine Gogendeau ${ }^{1}$, \\ Carole Pennetier ${ }^{1}$, and Renata Basto ${ }^{1} \mathbb{D}$
}

Ploidy variations such as genome doubling are frequent in human tumors and have been associated with genetic instability favoring tumor progression. How polyploid cells deal with increased centrosome numbers and DNA content remains unknown. Using Drosophila neuroblasts and human cancer cells to study mitotic spindle assembly in polyploid cells, we found that most polyploid cells divide in a multipolar manner. We show that even if an initial centrosome clustering step can occur at mitotic entry, the establishment of kinetochore-microtubule attachments leads to spatial chromosome configurations, whereby the final coalescence of supernumerary poles into a bipolar array is inhibited. Using in silico approaches and various spindle and DNA perturbations, we show that chromosomes act as a physical barrier blocking spindle pole coalescence and bipolarity. Importantly, microtubule stabilization suppressed multipolarity by improving both centrosome clustering and pole coalescence. This work identifies inhibitors of bipolar division in polyploid cells and provides a rationale to understand chromosome instability typical of polyploid cancer cells.

\section{Introduction}

Polyploidy is a condition in which the entire duplicated chromosome set is maintained within a single cell (Frawley and OrrWeaver, 2015). Polyploidy can be programmed and regulated. It is a strategy normally employed during development to increase metabolic potential through endoreplication (Orr-Weaver, 2015). Further, the increase in cell size through polyploidization can confer barrier functions essential during organogenesis (Unhavaithaya and Orr-Weaver, 2012). In these contexts, however, polyploid cells have limited proliferative capacity. In what appears somewhat paradoxical, events of unscheduled polyploidization, such as whole genome duplications (WGDs), have been identified in a variety of tumors and have been associated with chromosome instability (CIN) and poor prognosis (Bielski et al., 2018; Zack et al., 2013). Importantly, polyploid cells generated through cytokinesis failure give rise to tumors when transplanted subcutaneously into nude mice (Fujiwara et al., 2005), highlighting the transformation capacity of polyploid cells.

Concerning polyploidization through cytokinesis failure, it is important to take into account the increase in centrosome number. Each centrosome is composed of two centrioles surrounded by pericentriolar material (PCM), which is the site of microtubule
(MT) nucleation (Conduit et al., 2015). Centrosomes are normally duplicated only once during each cell cycle. This ensures that during mitosis, two centrosomes localized at opposite poles can sustain bipolar spindle assembly. In cells with supernumerary centrosomes, bipolar spindles can assemble thanks to centrosome clustering, an active process by which supernumerary centrosomes gather to form a single microtubule organizing center (MTOC; Basto et al., 2008; Ganem et al., 2009; Godinho et al., 2014; Kwon et al., 2008; Marthiens et al., 2012; Quintyne et al., 2005; Rhys et al., 2018; Silkworth et al., 2009). However, little is still known about centrosome clustering in cells that also contain increased DNA content (Dewhurst et al., 2014; Duncan et al., 2010; Fox et al., 2010; Storchova and Pellman, 2004).

Here we have characterized mitosis in polyploid cells generated through cytokinesis failure. We found that the accumulation of extra centrosomes and chromosomes leads to multipolarity in the majority of cases. Using genetic manipulations, laser ablation, and in silico approaches, we identified parameters that lead to multipolarity in polyploid cells. Interestingly, our work identifies MT stabilization as a suppressor of multipolarity, which might have important implications during tumorigenesis.

IInstitut Curie, Paris Science et Lettres Research University, Centre National de la Recherche Scientifique, Unité Mixte de Recherche UMR144, Biology of Centrosomes and Genetic Instability Laboratory, Paris, France; ${ }^{2}$ Center for Interdisciplinary Research in Biology, Collège de France, UMR7241/U1050, Paris, France; ${ }^{3}$ Department of Translational Research, Institut Curie, PSL University, Paris, France.

*A. Goupil and M. Nano contributed equally to this paper; Correspondence to Renata Basto: renata.basto@curie.fr; Maddalena Nano: mnano@ucsb.edu; M. Nano's present address is Molecular, Cellular, and Developmental Biology Department, University of California, Santa Barbara, Santa Barbara, CA.

(c) 2020 Goupil et al. This article is distributed under the terms of an Attribution-Noncommercial-Share Alike-No Mirror Sites license for the first six months after the publication date (see http://www.rupress.org/terms/). After six months it is available under a Creative Commons License (Attribution-Noncommercial-Share Alike 4.0 International license, as described at https://creativecommons.org/licenses/by-nc-sa/4.0/). 


\section{Results}

\section{Polyploid mitoses are multipolar}

Polyploidy can have important physiological roles in many animals and plants (Frawley and Orr-Weaver, 2015). It can be generated through different means such as endoreplication or endomitosis (Orr-Weaver, 2015). Here we focus our analysis of polyploid cells generated through cytokinesis failure. To characterize cell division in polyploid cells, we chose to use an in vivo model system that is permissive to cytokinesis failure, the Drosophila neural stem cells of the developing larval brain, also called neuroblasts (NBs; Gatti and Baker, 1989; Somma et al., 2002; Straight et al., 2005). We induced cytokinesis failure through Pavarotti (Pav; the Drosophila homologue of MKLP1; Adams et al., 1998) knock-down (KD) by inducible RNAi. Pav ${ }^{\mathrm{KD}}$ third instar brains revealed the presence of NBs of various sizes, indicative of different degrees of polyploidy, containing large amounts of DNA and increased centrosome numbers (Fig. 1, $\mathrm{A}-\mathrm{C}$ ). In this way, we generated polyploidy through cytokinesis failure in cells that are normally diploid. To facilitate comprehension, $\mathrm{Pav}^{\mathrm{KD}} \mathrm{NBs}$ will be referred simply as polyploid NBs.

To characterize mitosis by time-lapse microscopy, we used fly lines expressing transgenes encoding $a$-tubulin tagged with GFP (tubulin-GFP) and Histone H2Av variant tagged with RFP (histoneRFP). These allowed us to monitor spindle MTs and chromosomes, respectively. Control (Ctrl) diploid NBs divided asymmetrically, as described previously (Homem and Knoblich, 2012; IkeshimaKataoka et al., 1997; Fig. 1 D and Video 1). In polyploid NBs, several active MT-nucleating centrosomes were identified, and their number was increased in larger polyploid NBs (compare Fig. 1, E and F; and Video 1). Polyploid NBs presented multiple nuclei that entered mitosis in an asynchronous manner, as described using other genetic means of inducing polyploidy (Nano et al., 2019). After nuclear envelope breakdown (NEBD), extra centrosomes clustered in more than two groups, while chromosomes condensed and adopted a multilobed arrangement within a multipolar spindle, frequently centered within the cytoplasm. These multipolar configurations were never resolved into bipolar configurations, because multiple spindle poles failed to coalesce and were maintained as active MTOCs. Importantly, most polyploid anaphases were multipolar and generated several nuclei at mitotic exit (Fig. 1, E-G; and Video 1). These results are surprising, since in diploid NBs with centrosome amplification, induced through Sak, the PLK4 homologue, overexpression (SakOE), extra centrosomes always clustered in two major poles, and NBs invariably divided in a bipolar manner (Basto et al., 2006). The coalescence of spindle poles has been shown to favor the conversion of multipolar spindles into pseudo-bipolar or bipolar spindles in cancer cells (not polyploid) with extra centrosomes (Ganem et al., 2009; Silkworth et al., 2009). Together, our results suggest that bipolar spindle assembly in polyploid NBs, which requires a final step in spindle pole coalescence, is inhibited by the presence of extra DNA.

\section{Characterization of polyploid mitosis}

The analysis of polyploid NBs described above suggested a possible correlation between cell size and the degree of polyploidy. This was indeed the case, as DNA area correlated with cell area in polyploid NBs (Fig. 2 A). We next analyzed mitotic duration, defined as the time elapsed between NEBD and anaphase onset. We found that the large majority of polyploid NBs took more time to divide than Ctrl NBs (Fig. 2 B). Interestingly, this increased mitotic duration did not necessarily correlate with the degree of polyploidy. Small polyploid cells could take longer than larger cells to divide (Fig. 1, E and F; and Fig. 2 C). The increase in mitotic timing is a known feature of cells with extra centrosomes (Basto et al., 2008; Kwon et al., 2008), where it reflects a delay in satisfying the spindle assembly checkpoint (SAC), which monitors kinetochore-MT attachments (Musacchio, 2015). The observations reported here suggest that in polyploid cells, achieving accurate kinetochore-MT attachments takes longer than in diploid cells, independently of the degree of polyploidy.

We then wanted to assess the outcome of polyploid mitosis. While canonical cell division results in the separation of one nucleus in two daughter nuclei, polyploid mitosis frequently began with, and generated, multiple nuclei. To quantitatively assess the outcome of mitosis, we defined a parameter that we called nuclear index (NI). The NI was calculated as the ratio between the number of nuclei at anaphase and the number of nuclei at mitotic entry, divided by the number of daughter cells (see Materials and methods for details; Fig. 2 D). This allows us to distinguish between polyploid (only one daughter cell in our experimental setup) and diploid cells (two daughter cells). In diploid NBs, the NI was always 1, as NBs with one nucleus gave rise to two daughter NBs with one nucleus each after bipolar divisions. In contrast, in polyploid NBs that always started mitosis with at least two nuclei (generated through cytokinesis failure), the NI values spread between $<1$ and 4 . A NI $<1$ or $\mathrm{NI}=1$ reflected a reduction or maintenance in the number of nuclei at anaphase when compared with mitotic entry. However, the vast majority of cells displayed a NI $>1$, showing that, in a given cell, the number of nuclei increased at mitotic exit (Fig. 2, D and E). Interestingly, we noticed a positive correlation between the number of nuclei at mitotic exit and cell area (Fig. 2 F). Very large cells $\left(>4,000 \mu \mathrm{m}^{2}\right)$, which contained more centrosomes and chromosomes than smaller polyploid cells, generated a higher number of nuclei at mitotic exit than smaller polyploids. These results suggest that the degree of polyploidy impacts the outcome of nuclear divisions, with more nuclei being generated at each cell cycle.

\section{Probing the contribution of individual MT nucleation pathways to multipolarity in polyploid NBs}

At least three pathways contribute to MT nucleation during mitosis in animal cells (Hayward et al., 2014; Prosser and Pelletier, 2017). We hypothesized that the multipolar outcome typical of polyploid NB mitosis could result from an excess of MTs nucleated from extra centrosomes, chromosomes, or preexisting MTs. We tested their contribution by decreasing the expression of key players in each pathway individually.

We first ascertained the contribution of the centrosomal pathway. To reduce centrosome-dependent MT-nucleation, we used a mutation in the sas-4 gene (Centrosomal P4.1-associated protein in humans), which encodes an essential centriole duplication gene (Basto et al., 2006; Kirkham et al., 2003). Small to medium size polyploid, sas4 ${ }^{m u t}$ NBs started by assembling an amorphous MTstructure around the DNA. Surprisingly, this structure evolved into an almost perfect bipolar spindle, forming an array similar to the 
A

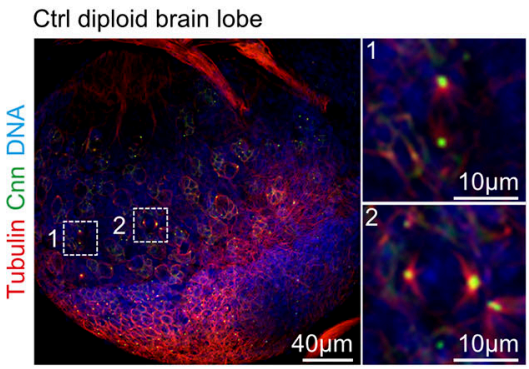

D

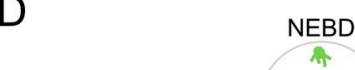

Ctrl

Diploid NB

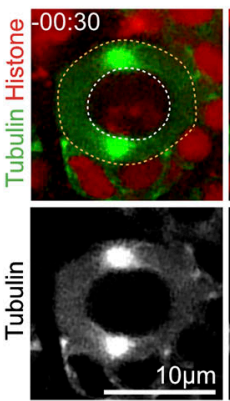

so
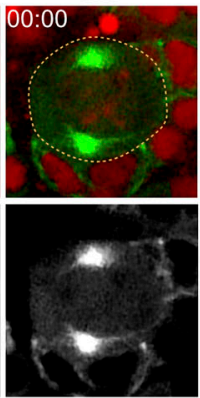

B

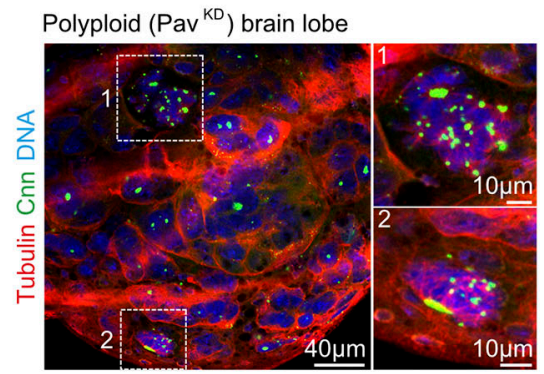

Prometaphase

皮

Metaphase
C

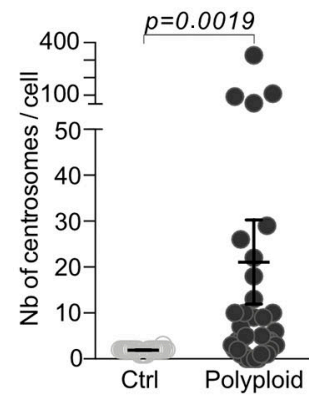

E

Asynchronous NEBD

Multipolar spindle

Bipolar anaphase

x

$\frac{\mathbb{N}}{\mathrm{W}} \rightarrow$ 似
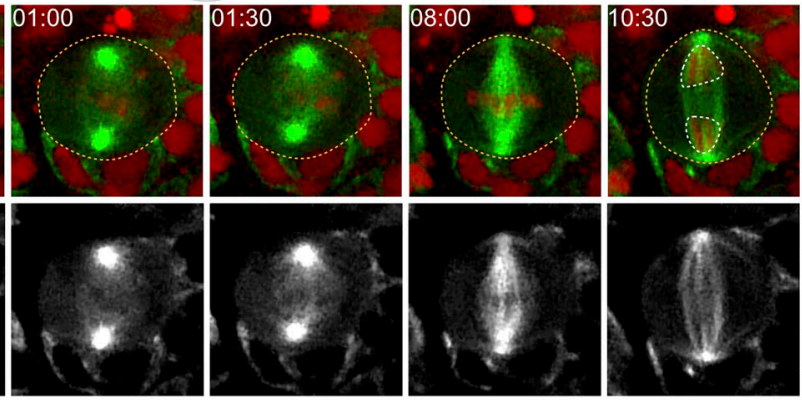

$\mathrm{Pav}^{\mathrm{KD}}$

Small polyploid NB

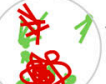

Initial
centrosome
clustering
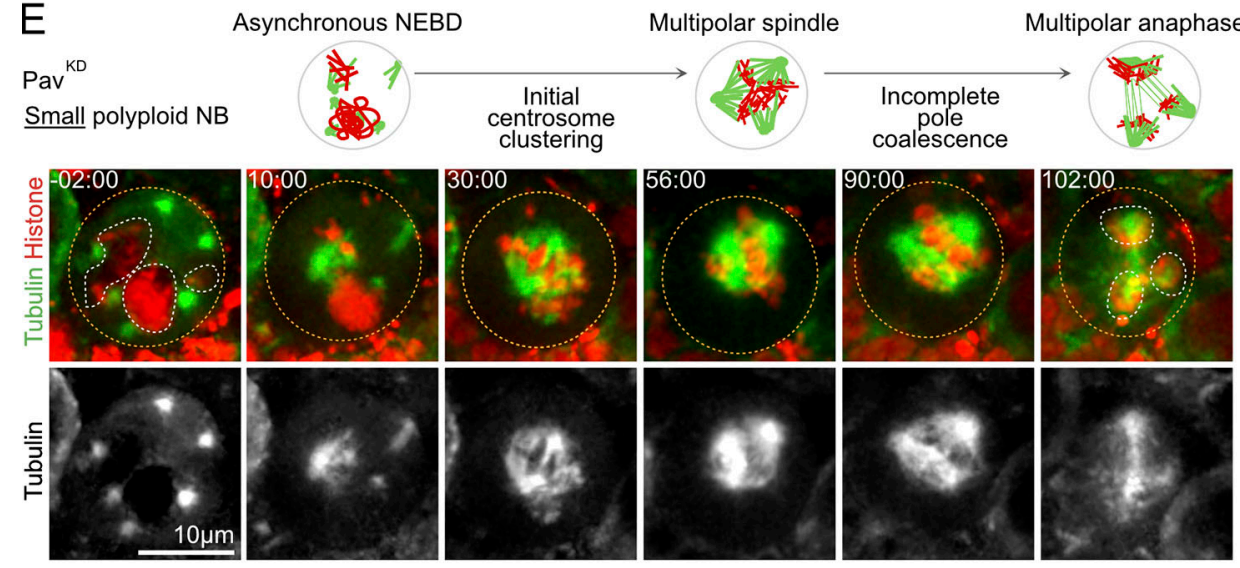

Asynchronous NEBD

Multiple mini-spindles
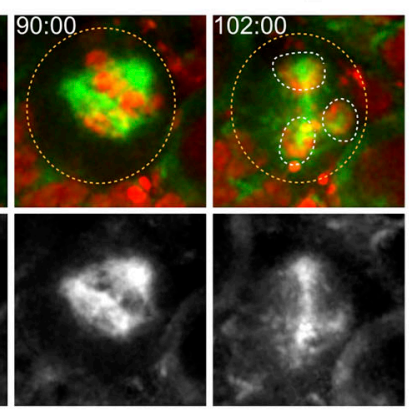

F
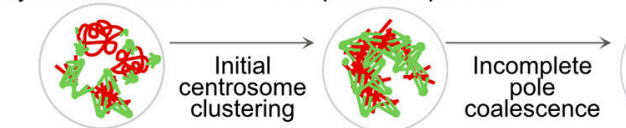

Pole sorting Multipolar anaphase

G
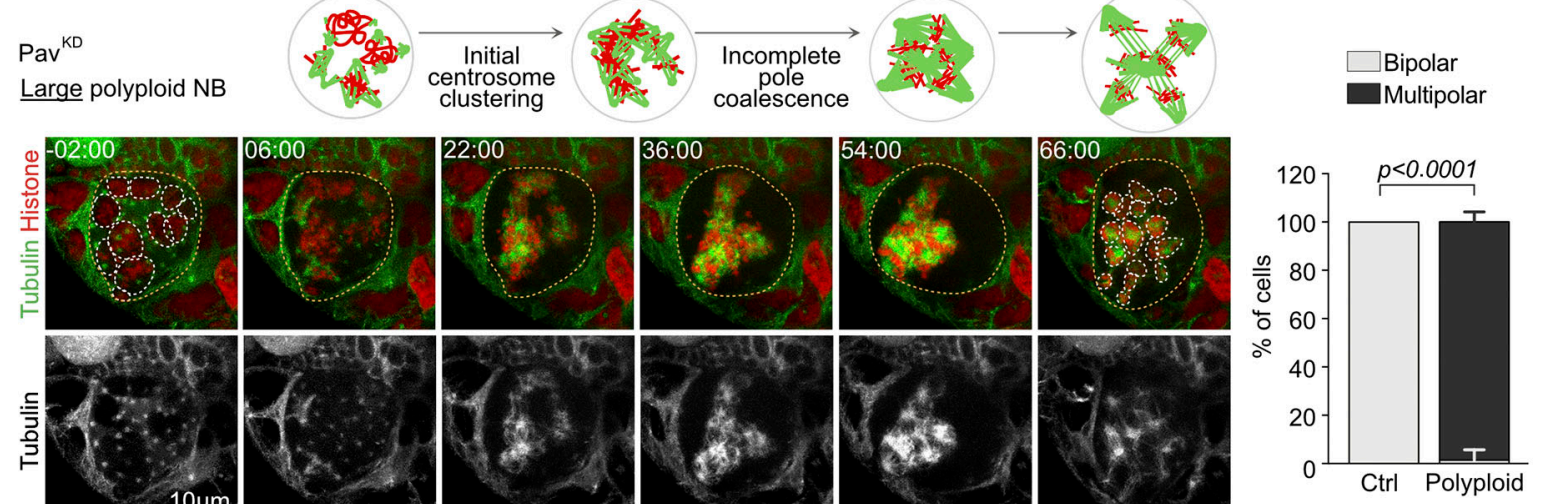
Figure 1. Polyploid NBs undergo multipolar mitosis. (A and B) Images of whole mount diploid (A) and polyploid (B) brain lobes (BL) and NBs (insets) labeled with antibodies against a-tubulin (red) and $\mathrm{Cnn}$ (green). DNA in blue. (C) Dot plot of centrosome number (Nb) per Ctrl $(n=30 \mathrm{NBs}$ from 4 BL) and polyploid ( $n=$ $38 \mathrm{NBs}$ from $10 \mathrm{BL}$ ) NBs. Statistical significance was determined using a $t$ test. (D-F) Stills of time-lapse videos of mitotic NBs expressing tubulin-GFP (green and gray in the bottom insets) and histone-RFP (red). Orange and white dotted circles surround cells and nuclei, respectively. Time of mitosis is indicated in minutes:seconds. Time 00:00 corresponds to NEBD. Schematic representations above the stills. (D-F) Ctrl diploid (D), small (E), and large (F) polyploid NBs. (G) Percentage of cells in each category in Ctrl ( $n=34 \mathrm{NBs}$ from $2 \mathrm{BL}$ ) and polyploid NBs $(n=107 \mathrm{NBs}$ from $37 \mathrm{BL})$. Statistical significance by a multiple $t$ test. Error bars represent the mean \pm SD and $p$ the $P$ value.

one observed in diploid, sas4 ${ }^{\text {mut }}$ NBs, albeit larger (Fig. 3 A, Fig. S1 A, and Video 2). However, the ability of centrosome loss to sustain bipolar spindle assembly was limited by cell size. Medium or large polyploid, sas $4^{m u t} \mathrm{NBs}$ divided in a multipolar manner even in the absence of centrosomes or when centrosome number was highly reduced (Fig. 3, B and C; and Video 2). Mitotic timing was decreased in polyploid, sas4 ${ }^{m u t}$ NBs when compared with polyploid NBs (Fig. $3 \mathrm{D}$ and Table S1). Consistent with a decrease in multipolarity, the number of nuclei generated at anaphase was lower but still correlated with cell area (Fig. 3 E). Further, polyploid, sas4 ${ }^{m u t}$ NBs presented a high proportion of cells with a NI $\leq 1$ (Fig. $3 \mathrm{~F}$ ), confirming that a reduction in centrosome number in polyploid cells promotes the generation of fewer nuclei at mitotic exit. We concluded that MT nucleation from the centrosomes contributes to the assembly of multipolar spindles in polyploid NBs.
In contrast to the centrosomal pathway, reducing the Augmin or chromatin-mediated MT-nucleation pathways (CMP) did not reduce multipolarity. We used previously validated RNAi tools to deplete Mars and the Augmin Dgt2 subunit (Goshima et al., 2008; Hayward et al., 2014). Polyploid, Mars ${ }^{\mathrm{KD}}$ and polyploid, Aug $^{\mathrm{KD}}$ NBs always divided in a multipolar fashion, while respective diploid conditions always divided in a bipolar manner (Fig. S1, B-F; and Videos 3 and 4). Manipulation of either of these pathways did not reduce the NI in polyploid NBs but impacted the mitotic timing in opposite directions. The mitotic timing was reduced in polyploid, Mars ${ }^{\mathrm{KD}}$, while it increased in polyploid, AugKD (Fig. S1, G-I). Interestingly, in certain polyploid, Mars ${ }^{\mathrm{KD}}$ NBs and polyploid, AugKD NBs, we noticed the presence of several individual spindles that assembled between the main spindle and the cell cortex (Fig. S1, C and E, white arrows). These
A

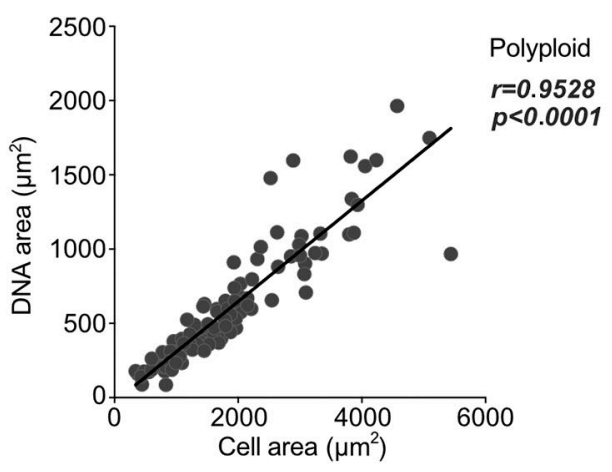

D

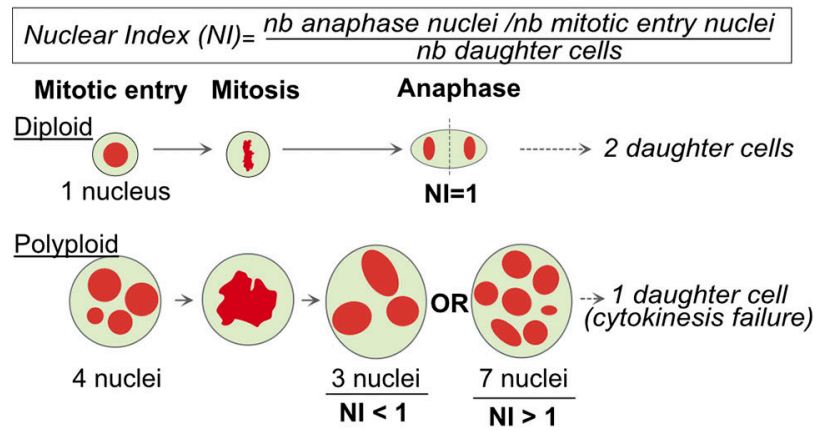

$\mathrm{B}$

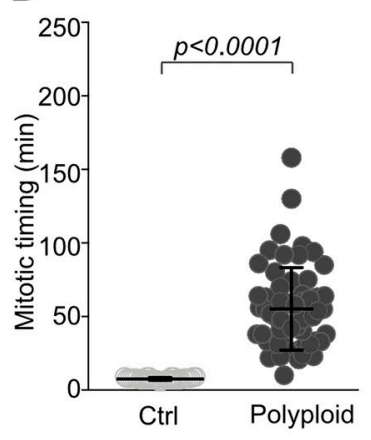

E

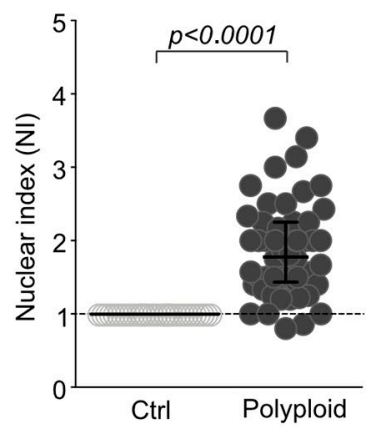

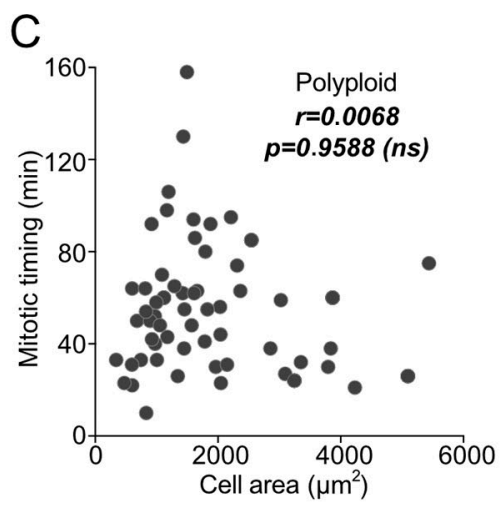

$\mathrm{F}$

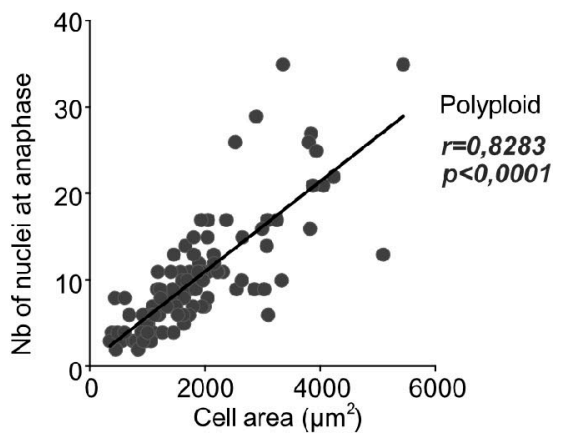

Figure 2. Characterization of polyploid mitosis in Drosophila NBs. (A) XY plot representing DNA and cell area in polyploid NBs ( $n=112$ NBs from 37 BL). (B) Dot plot showing the time spent in mitosis for $\mathrm{Ctrl}(n=34 \mathrm{NBs}$ from $2 \mathrm{BL}$ ) and polyploid ( $n=60 \mathrm{NBs}$ from $31 \mathrm{BL}) \mathrm{NBs}$. (C) XY plot representing the mitotic timing and cell area ( $n=60 \mathrm{NBs}$ from $31 \mathrm{BL})$. (D) Schematic representation of NI calculation. (E) Dot plot of NI in Ctrl $(n=34 \mathrm{NBs}$ from $2 \mathrm{BL})$ and polyploid NBs $(n=54 \mathrm{NBs}$ from $28 \mathrm{BL}$ ). (F) XY plot of nuclei at anaphase and cell area ( $n=107 \mathrm{NBs}$ from $37 \mathrm{BL})$. (B and E) Statistical significance by a Mann-Whitney test. Error bars represent the mean \pm SD (B) and the median \pm interquartile range (E). (A, C, and F) Statistical significance of the correlation by a Spearman $r$ test. ns, not significant; $p, P$ value; $r$, correlation coefficient; $\mathrm{Nb}$, number. 
A

Small polyploid, sas $4^{\text {mut }}$

Bipolar division

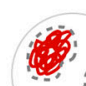

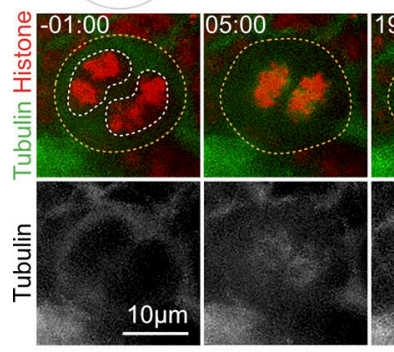

C

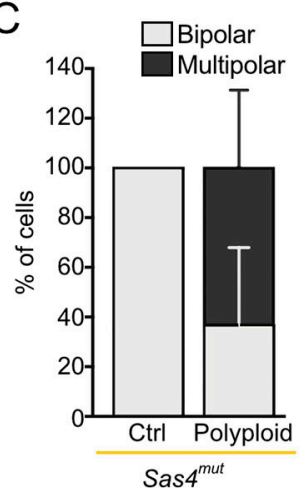

B Large polyploid, sas $4^{\text {mut }}$ Multipolar division
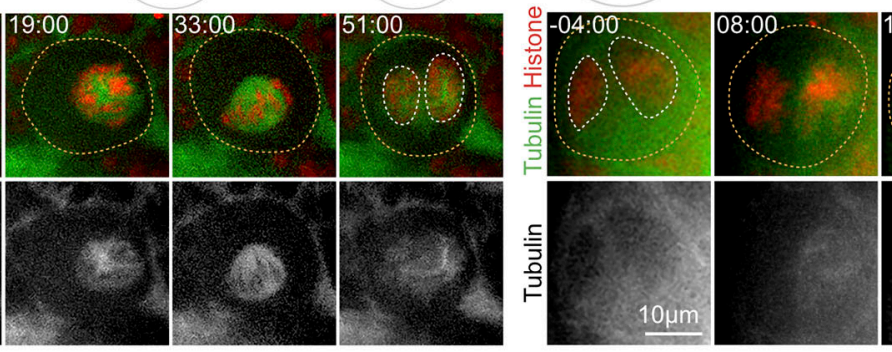

Multipolar spindle

Multipolar anaphase
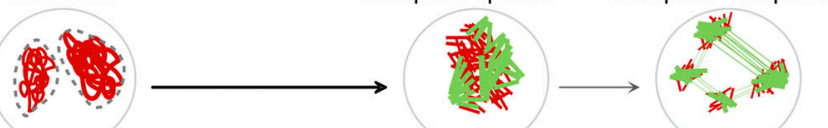

E
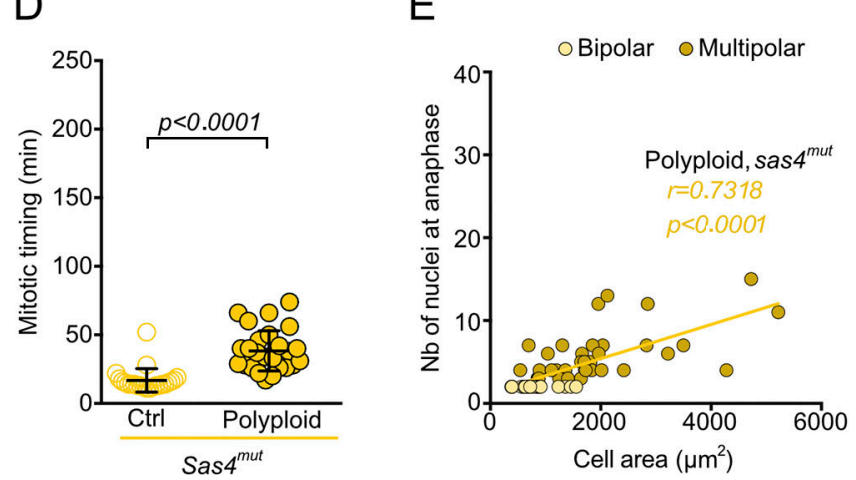

$\mathrm{F}$

Figure 3. Small polyploid NBs without centrosomes divide bipolarly. (A and B) Stills of time-lapse videos of mitotic small (A) and large (B) polyploid, sas4mut NBs expressing tubulin-GFP (green and gray in the bottom insets) and histone-RFP (red). Orange and white dotted circles surround cells and nuclei, respectively. Time of mitosis is indicated in minutes:seconds. Time 00:00 corresponds to NEBD. Schematic representations of mitosis above the stills. (C) Graph bars showing the percentage of cells in each category in Ctrl, sas4 $4^{\text {mut }}\left(n=23 \mathrm{NBs}\right.$ from $5 \mathrm{BL}$ ), and polyploid, sas4 $4^{\text {mut }}(n=56$ NBs from $22 \mathrm{BL}) \mathrm{NBs}$. (D) Dot plot showing the time spent in mitosis for Ctrl, sas4 $4^{\text {mut }}$ ( $n=23 \mathrm{NBs}$ from $5 \mathrm{BL}$ ), and polyploid, sas4 ${ }^{\text {mut }}$ ( $n=28 \mathrm{NBs}$ from $14 \mathrm{BL}$ ) NBs. (E) XY plot of nuclei at anaphase and cell area ( $n=56 \mathrm{NBs}$ from $22 \mathrm{BL}$ ). Statistical significance of the correlation by a Spearman $r$ test, $r$ corresponds to the correlation coefficient. (F) Dot plot of $\mathrm{NI}$ in Ctrl, sas $4^{\text {mut }}\left(n=23 \mathrm{NBs}\right.$ from $5 \mathrm{BL}$ ), and polyploid, sas $4^{m u t}$ ( $n=28 \mathrm{NBs}$ from $\left.14 \mathrm{BL}\right) \mathrm{NBs}$. (D and F) Statistical significance by a Mann-Whitney test. Error bars represent the mean \pm SD (D) and the median \pm interquartile range (F), $p$ the $P$ value. ns, not significant.

results show that the centrosomal pathway is the major contributor to multipolarity in polyploid mitosis.

\section{Centrosome clustering takes place in polyploid mitosis, but it} is not sufficient to promote bipolar spindle assembly

In cells with extra centrosomes, the minus-end directed kinesin Ncd (Drosophila orthologue of HSET/ KIFC1) plays an essential role in centrosome clustering (Basto et al., 2008; Kwon et al., 2008; Rhys et al., 2018). We induced polyploidy in ncd mutants (ncd ${ }^{m u t}$; Endow and Komma, 1998) and readily noticed that after NEBD, in polyploid, $n c d^{m u t}$ NBs, centrosomes did not cluster. Instead, they formed individual and independent poles, leading to the formation of a network of multiple mini-spindles, which remained connected to each other. As cells progressed through mitosis, this multipolar status was maintained, and nuclear divisions were always multipolar, while mitoses were always bipolar in diploid, $n c d^{m u t}$ NBs (Fig. S2, A, B, and E; and Videos 3 and 4). Although mitotic duration was increased in polyploid, $n d^{m u t}$ NBs compared with diploid, $n c d^{m u t}$, it was similar to polyploid NBs (Fig. S2 F and Table S1), suggesting that failure in the initial centrosome clustering step does not delay mitotic progression.
We reasoned that, in polyploid NBs, Ncd might be a limiting factor precluding pole coalescence into two main MTOCs. To test this possibility, we overexpressed Ncd (NcdOE). Diploid, NcdOE cells divided normally (Fig. S2 C and Video 3). However, multipolarity was maintained in polyploid, NcdOE NBs (Fig. S2, D and E; and Video 4). In both polyploid, ncd ${ }^{m u t}$ and polyploid, NcdOE NBs, the number of nuclei at anaphase correlated with cell size. Importantly, a higher number of nuclei at anaphase was noticed in polyploid, $n c d^{m u t} \mathrm{NBs}$ when compared with polyploid NBs even for cells of the same size (Fig. S2 G and Fig. 2 F), confirming the increase in multipolarity when Ncd is absent. Importantly, the NI in polyploid, ncd ${ }^{m u t}$ NBs and polyploid, NcdOE was mostly $>1$ (Fig. S2 H). These results suggest that Ncd plays an essential role in the initial centrosome clustering step in polyploid mitosis. Further, they suggest that the second step of spindle pole coalescence is not limited by insufficient Ncd levels.

\section{Centrosome number, DNA content, and chromosome shape influence the final step of spindle pole coalescence in polyploid cells}

To further characterize spindle pole coalescence in polyploid NBs, we used Plp and Cnn to label centrioles and PCM. Mitotic 
polyploid NBs contained condensed chromosomes arranged in a multilobed configuration separating large spindle poles, which could be visualized as large $\mathrm{Cnn}$ structures containing multiple Plp-positive dots (Fig. 4, A and B). We filmed brains expressing Histone-RFP and Spd2-GFP to label centrosomes. At mitotic entry, several centrosomes clustered in multiple poles, consistent with our previous observation that, by late prometaphase, each pole contained several clustered centrosomes. However, these poles did not coalesce, but remained separated by condensed chromosomes until anaphase onset, which was always multipolar (Fig. 4, C and D; and Video 5).

To identify the contributors of multipolarity and lack of spindle pole coalescence in polyploid cells, we established an in silico approach. We designed simulations using Cytosim, a cytoskeleton-dedicated agent-based software package (Nedelec and Foethke, 2007; see Materials and methods and Table S2 for details). Mitotic cells were considered as circular shapes (2D) with confined MTs, DNA, and motors. With the aim of validating the in silico approach, we configured a system where the number of centrosomes, modeled as asters nucleating MTs radially, was increased in conditions of fixed DNA content, modeled as mobile beads clustered as nuclei. At the start of the simulations, centrosomes were randomly positioned while DNA clusters were slightly centered. In the presence of a constant and high DNA level, simulations containing 10 centrosomes were mostly resolved in a bipolar spindle. However, an increase in centrosome number above a certain threshold highly reduced the bipolar outcome in favor of multipolar spindles (Fig. 4, E and F; and Video 6). This is in line with our observations in polyploid, sas4 ${ }^{m u t} \mathrm{NBs}$, as a decrease in centrosome number favored the formation of bipolar spindles. Next, we tested the consequences of DNA levels variation to a polyploid-like status (from 9 to 41 DNA beads), in conditions of high (30) centrosome number. In simulations containing $\leq 17$ beads, spindles were mostly bipolar. However, mitotic spindles became multipolar just by increasing the number of beads (Fig. 4, G and H; and Video 6). These results can be compared with what has been described in SakOE (Basto et al., 2008) and polyploid NBs (this work): increase in DNA content in the presence of extra centrosomes results in multipolar divisions, while centrosome amplification is permissive to bipolar divisions if the DNA levels are not considerably increased.

We then calculated the DNA shape aspect ratio (defined as the ratio between width and length of the chromosomes plate at the end of the simulation) and found that DNA beads could be organized in classic "metaphase-like" shapes (aspect ratio closer to 0 ) or "cross-like" shapes (aspect ratio closer to 1). Interestingly, the increase in the DNA shape aspect ratio correlated with multipolarity (Fig. $4 \mathrm{I}$ ). This result prompted us to test if abnormal DNA configurations, such as the metaphase-like figures seen in vivo in polyploid NBs (Fig. 1, E and F; and Fig. 4, B and D), influence spindle multipolarity. We used a minimalist system where the DNA is represented as immobile metaphase-like plates displaying different morphologies. While centrosomes clustered into two main poles when the DNA shape corresponded to a classical metaphase plate, simulations with more complex DNA shapes always resulted in multipolar spindles
(Fig. 4, J and K; and Video 6). These observations suggest that DNA content, and in particular the chromosome configurations, influences spindle pole coalescence and leads to multipolarity in silico.

Since the simulations exposed a contribution of extra DNA to inhibit spindle pole coalescence, we turned to in vivo experiments to confirm these findings. We tried to reduce DNA content of polyploid NBs using DNase treatment or laser ablation. Unfortunately, these were highly toxic (data not shown). To overcome this drawback, we designed an alternative strategy to induce multipolarity independently of polyploidy. We used a combination of SakOE with mutations in the mad2 gene. Mad2 is a member of the SAC (Musacchio and Hardwick, 2002), and a fraction of SakOE, mad2 ${ }^{m u t} \mathrm{NBs}$ divide multipolarly and generate aneuploid daughter cells due to defects in centrosome clustering (Basto et al., 2008; Gogendeau et al., 2015; Kwon et al., 2008). Analysis of mitotic SakOE, mad2 $2^{m u t}$ NBs by time-lapse microscopy revealed that in certain NBs, chromosomes were positioned in a way that seemed to prevent centrosome clustering and pole coalescence (Fig. S3, A-E). When the DNA was ultimately repositioned toward the metaphase plate, a bipolar spindle was assembled. A complete or partial lack of spindle pole coalescence was observed if one or multiple chromosomes were maintained between two poles. These observations strengthen our hypothesis and support a model in which chromosomes act as a barrier to spindle pole coalescence. They also suggest that the barrier size might ultimately determine whether spindle poles will coalesce or not.

\section{Chromosomes act as a barrier to spindle pole coalescence in human cancer cells}

The results obtained in Drosophila NBs showed that extra DNA of polyploid cells can act as a barrier to spindle bipolarity. We wanted to test if this was also the case in the ovarian cancer cell line OVCAR-8, since multipolar divisions might have deleterious consequences on genetic stability. We generated doxycycline (Dox)-inducible OVCAR-8 cell lines that overexpress the Sak homologue, PLK4 (hence referred to as PLK4OE cells), and stably express the H2B-RFP to test their clustering capacity. Characterization of the OVCAR-8 PLK4OE cell line showed that at least $56 \%$ of the cells contained extra centrosomes (Fig. S4, A and B). Importantly, these cells, incubated with SiR-tubulin to follow the spindle, by time-lapse microscopy, divide bipolarly (Fig. 5, $\mathrm{A}-\mathrm{C}$; and Video 7). Even if chromosome barriers were noticed, these appear rather small and were resolved during progression through prometaphase, allowing spindle pole coalescence (Fig. 5 B, white arrowhead and arrows).

To generate polyploid OVCAR-8 cells, we inhibited cytokinesis in a population of asynchronous cells using a short (1-h) pulse of Latrunculin B (LatB), an actin polymerization inhibitor. Cells were then released for $24 \mathrm{~h}$ before analysis. Immunostaining of Ctrl (EtOH-treated) or polyploid (LatB-treated) OVCAR-8 cells clearly showed an enrichment of multipolar and pseudo-bipolar spindles containing a DNA barrier (Fig. 6, A and B). Interestingly, we observed that certain spindle poles in these cells contained more than one centrosome (Fig. 6 B), showing that in polyploid OVCAR-8 cells, similarly to 
A

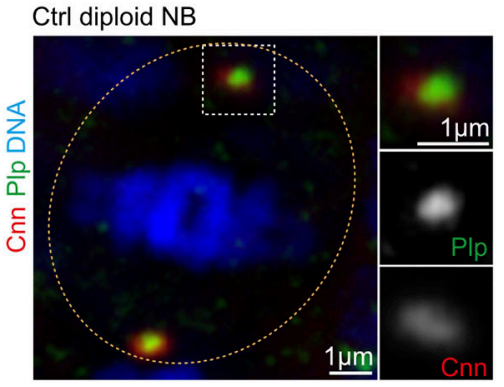

C

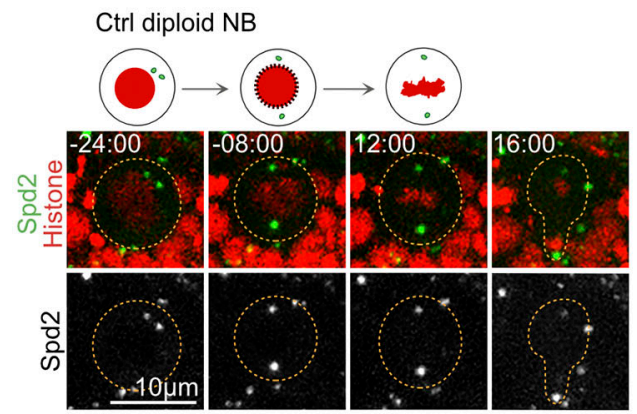

E

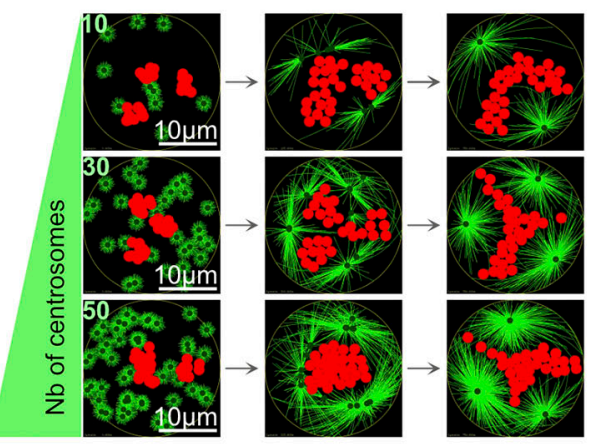

G

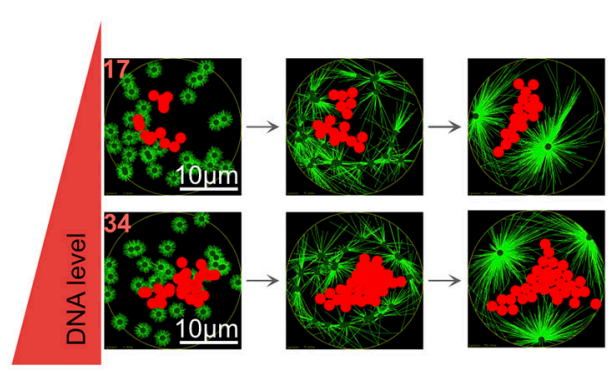

$J$

DNA shape 1: "metaphase-like"

DNA shape 2: "3-pointed star" "4-pointed star"

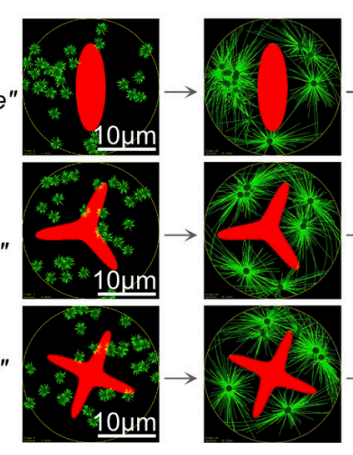

B

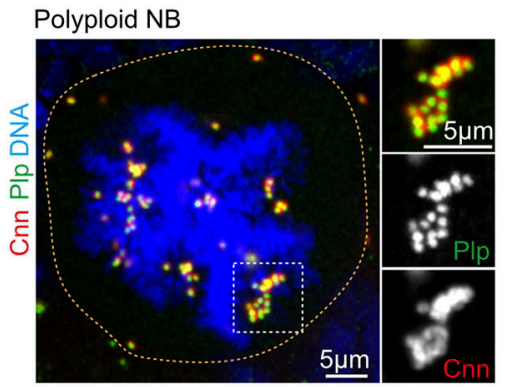

D Polyploid NB
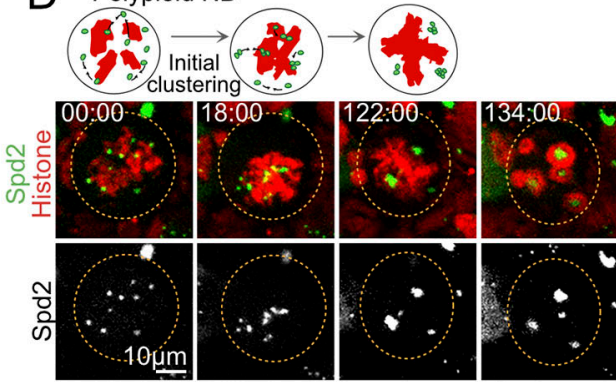

$\mathrm{F}$
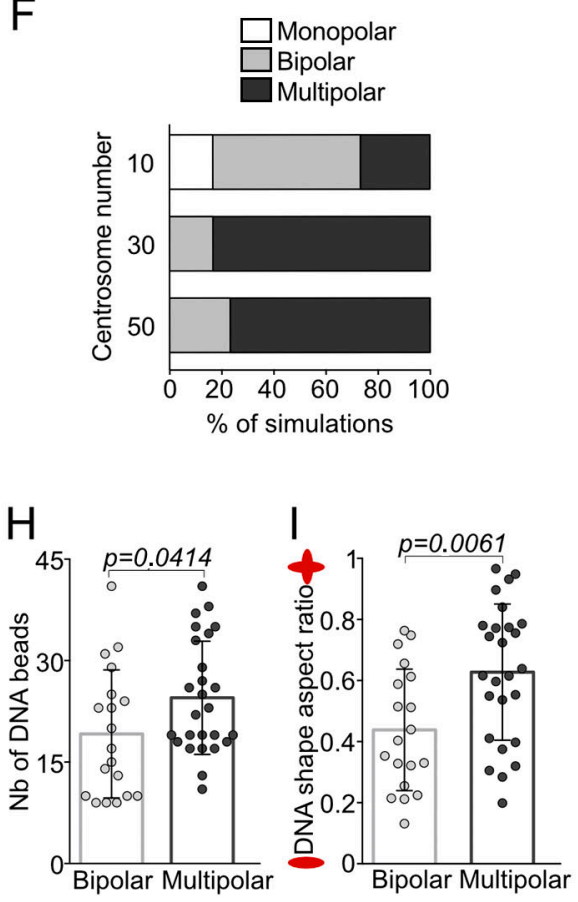

K

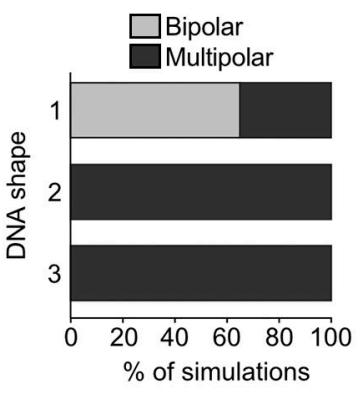


Figure 4. Centrosome number, DNA content, and shape influence mitotic spindle assembly. (A and B) Images of diploid (A) and polyploid (B) NBs labeled with Plp (green and gray) and Cnn (red and gray) antibodies. DNA in blue. (C and D) Stills of time-lapse videos of mitotic diploid (C) and polyploid (D) NBs expressing Histone 2B-RFP (red) and Spd2-GFP (green and gray in the bottom insets), corresponding to DNA and centrosomes. Time of mitosis is represented in minutes:seconds. Time 00:00 corresponds to NEBD. Schematic representations of mitosis above the stills. (A-D) Orange circles highlight NBs. (E, G, and J) Representative images of Cytosim-based simulations of spindle assembly around the DNA represented as mobile beads (E and $\mathrm{G}$ ) or immobile plate (J). DNA is in red; MTs, green lines; and centrosomes, green circles. (E) Simulations with fixed DNA content and variable number of centrosomes. (F) Graph bars showing the percentage of simulations in each category $(n=30$ simulations/condition). (G) Simulations with fixed number of 30 centrosomes and increasing number of mobile DNA beads. ( $\mathbf{H}$ and $\mathbf{I})$ Dot plots representing the number of DNA beads $(\mathrm{H})$ and DNA shape aspect ratio $(\mathrm{I})$ in simulations with bipolar or multipolar status ( $n=45$ simulations). Statistical significance was determined using a Mann-Whitney test. Error bars represent the mean \pm SD and $p$ the $P$ value. (J) Simulations with fixed number of 30 centrosomes and different DNA shape configurations. (K) Graph bars of the percentage of simulations in each category ( $n=20$ simulations/condition).

Drosophila polyploid NBs, centrosome clustering can take place, but not spindle pole coalescence. To explore the dynamics of cell division in this system by time-lapse microscopy, we generated cell lines stably expressing H2B-GFP that were incubated with SiR-tubulin, allowing us to follow chromosomes and spindles, respectively. Strikingly, the presence of chromosomes lingering between two spindle poles was readily observed in polyploid cells. Importantly, these cells mostly divided in a multipolar fashion (Fig. 6, C-E; and Video 7). Interestingly, when we measured the area of chromosome barriers before

A Control OVCAR-8 cells (DOX-)
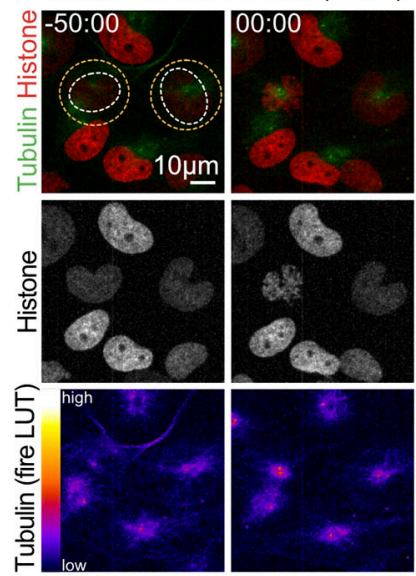

$\mathrm{B}$ PLK4OE OVCAR-8 cells (DOX+)
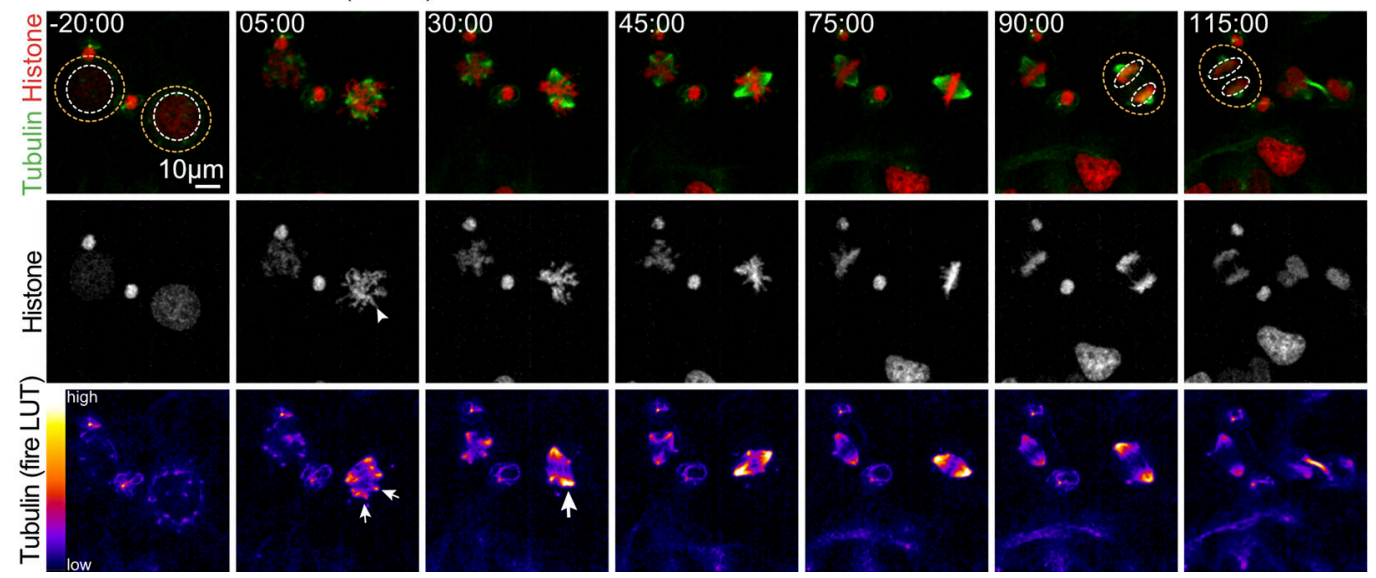

C

Figure 5. OVCAR-8 cells are proficient for centrosome clustering. (A and B) Stills of time-lapse videos of mitotic Ctrl (DOX-; A) and centrosome amplified PLK4OE (DOX+; B) OVCAR-8 cells expressing Histone 2B-RFP (red and gray in the middle insets) and incubated with SiR-tubulin (green and fire lookup table; LUT in the bottom insets). Orange and white dotted circles surround cells and nuclei, respectively. Time of mitosis is represented in minutes:seconds. Time 00: 00 corresponds to the first NEBD. (C) Graph bars showing the percentage of cells in C $\operatorname{trl}(n=149$ cells from two independent experiments) and PLK4OE ( $n=161$ cells from two independent experiments) OVCAR-8 cells. Statistical significance by a multiple $t$ test. Error bars represent the mean \pm SD, $p$ the P value; ns, not significant. 
A

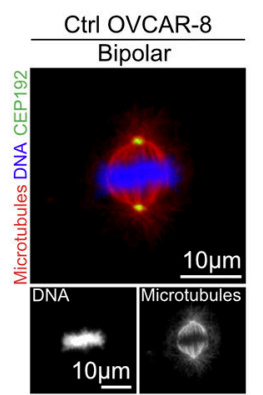

B

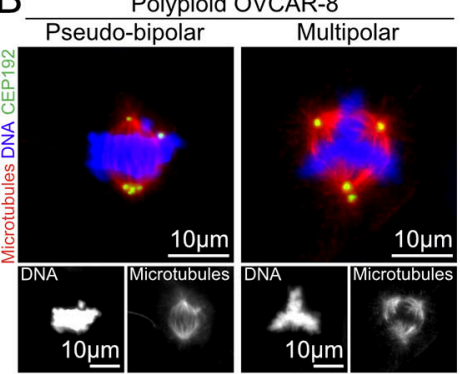

C Binucleated polyploid OVCAR-8 cell - resolved small chromosome barrier = bipolar
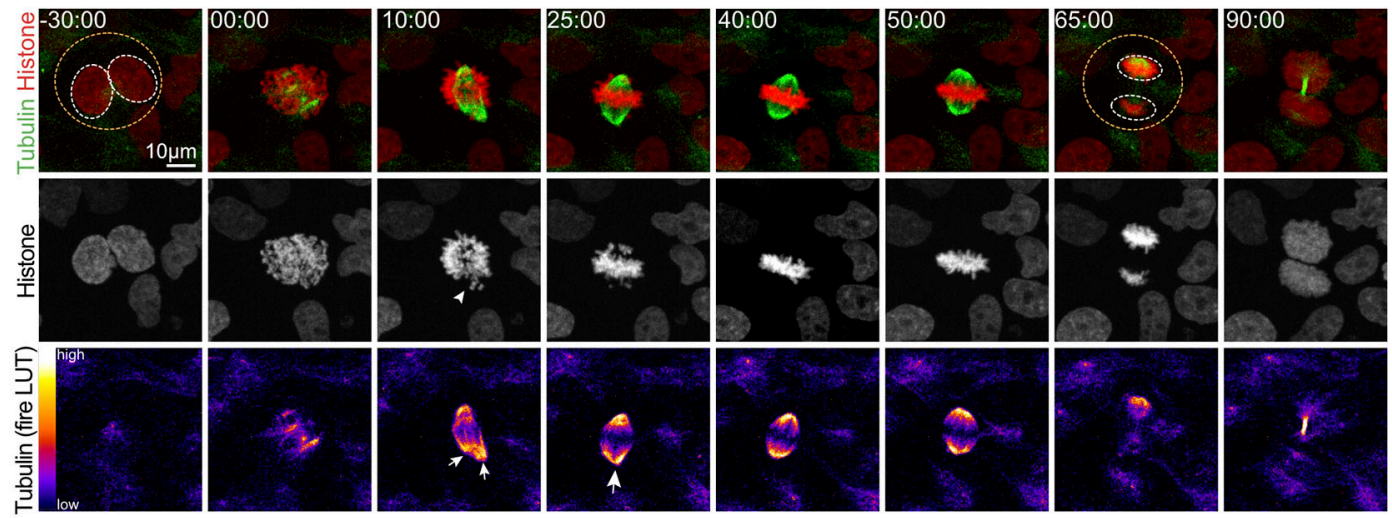

D

Binucleated polyploid OVCAR-8 cell - unresolved large chromosome barrier = multipolar
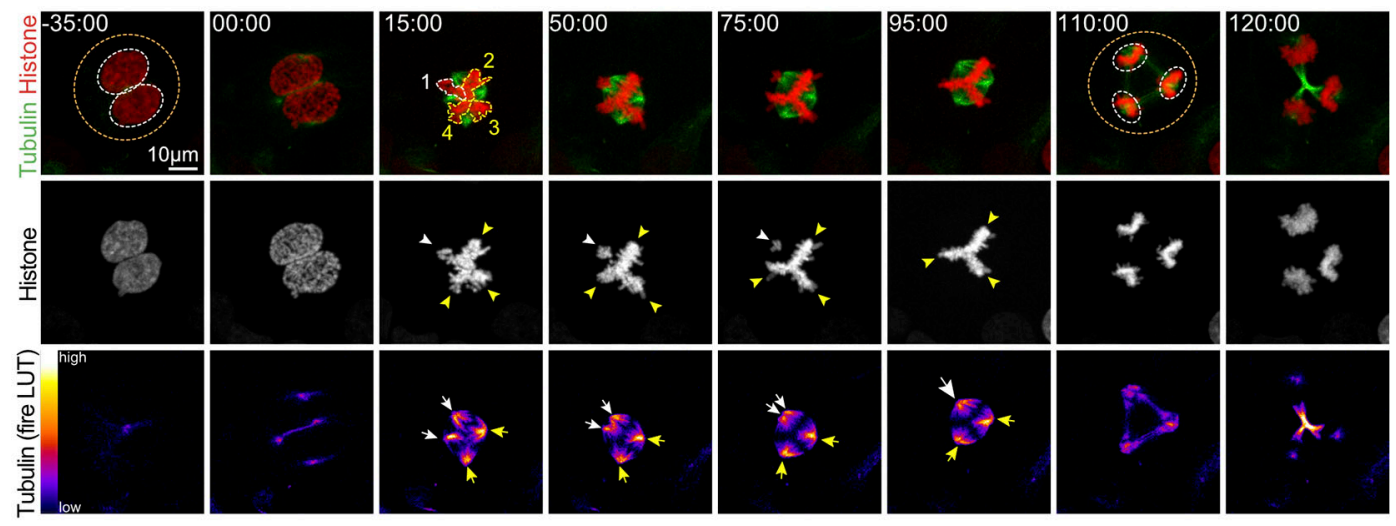

E

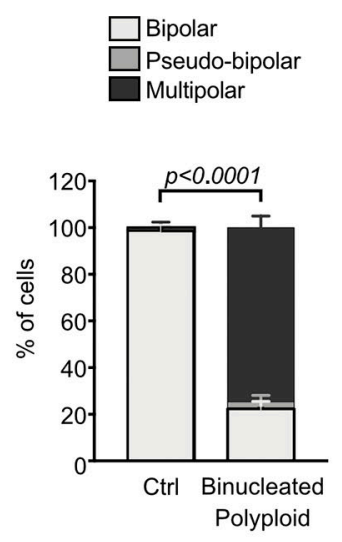

$\mathrm{F}$

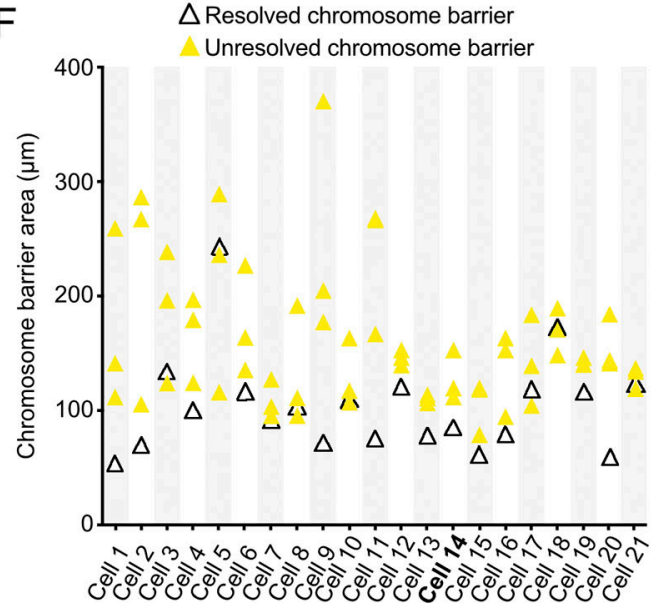


Figure 6. Polyploid OVCAR-8 cells also divide multipolarly and fail spindle pole coalescence. (A and B) Images of Ctrl (A) and polyploid (B) OVCAR8 cells labeled with antibodies against a-tubulin (red and gray) and CEP192 (green) to label the mitotic spindle and the PCM, respectively. DNA in blue and gray. (C and D) Stills of time-lapse videos of mitotic binucleated polyploid OVCAR-8 cells expressing Histone 2B-GFP (red and gray in the middle insets) and incubated with SiR-tubulin (green and fire LUT in the bottom insets). Orange and white dotted circles surround cells and nuclei, respectively. Time of mitosis is represented in minutes:seconds. Time 00:00 corresponds to NEBD. (E) Graph bars of the percentage of cells in each category in Ctrl ( $n=142$ cells from three independent experiments) and binucleated polyploid OVCAR-8 cells $(n=101$ cells from three independent experiments). Statistical significance by a multiple $t$ test. Error bars represent the mean \pm SD and $p$ the P value between "multipolar" populations. (F) Dot plot showing the area of revolved (white) and unresolved (yellow) chromosome barriers in 21 polyploid OVCAR- 8 cells. Stills of cell 14 (bold) shown in D.

spindle pole coalescence, we noticed that in most cases, the barrier displaying the smallest area was the one resolved, while the remaining larger DNA barriers represented obstacles to coalescence (Fig. 6, C, D, and F, white and yellow arrowheads).

To directly demonstrate the function of chromosome barriers in inhibiting spindle pole coalescence, we used laser ablation to remove chromosome(s) that were positioned between two poles. Remarkably, this led to the coalescence of spindle poles previously separated by the chromosome barrier in $53 \%$ of the cases ( $n=39 / 74$ cells; Fig. 7, A, B, and E; and Video 8). We also observed that in $47 \%$ of the cases that did not coalesce $(n=35 / 74$ cells), this was explained in $19 \%$ of the cases ( $n=14 / 35$ cells) by the remodeling of the chromosomes within the abnormal prometaphase plate, which generated a second chromosome barrier (Fig. 7 C). In the remaining $28 \%(n=21 / 35$ cells), no further chromosome barriers were formed after ablation. However, we noticed that unclustered poles were positioned far away from each other (Fig. 7 D). Recently, it has been shown that HSET/ KIFC1, the human Ncd homologue, requires a minimal pole distance to trigger efficient clustering (Rhys et al., 2018). Therefore, we measured pole-to-pole distance just before laser ablation. Interestingly, a lack of coalescence was noticed in conditions with large pole-to-pole distances $(8.11 \pm 2.36 \mu \mathrm{m})$. In contrast, this distance was smaller in situations where coalescence occurred $(6.02 \pm 1.72 \mu \mathrm{m} ; \mathrm{P}=0.0002$; Fig. $7 \mathrm{~F})$. These results suggest that chromosomes can indeed act as a barrier to spindle pole coalescence. If this barrier is removed, a permissive HSET/KIFC1 working distance between the two poles must be reached to generate efficient coalescence.

\section{Mononucleated polyploid cells also divide multipolarly}

So far, our work showed that binucleated OVCAR-8 cells and multinucleated polyploid Drosophila NBs assemble multipolar spindles, since centrosomes and poles fail to cluster due to the presence of chromosome barriers. To test if chromosome barriers and subsequent multipolarity are less likely to be formed when a single nucleus was present at mitotic entry, we generated simulations where the 30 DNA beads were distributed in one or three groups (representing nuclei) in the presence of 30 centrosomes. In either case, the percentage of multipolarity was comparable (Fig. 8, A and B; and Video 9), suggesting that extra DNA (rather than its organization in multiple nuclei) favors the formation of chromosome barriers.

We next attempted to generate Drosophila mononucleated polyploid NBs through endoreplication. Unfortunately, these cells only rarely entered mitosis, precluding the analysis of mitotic progression (data not shown). Thus, we generated mononucleated polyploid OVCAR-8 cells through the combination of Eg5 and MPS1 inhibition. Inhibition of Eg5 with monastrol generates monopolar spindles that arrest in a prometaphase-like state (Kapoor et al., 2000). MPS1 inhibition relieves the SAC and forces arrested cells to exit mitosis even without anaphase (Santaguida et al., 2010), generating a high frequency of polyploid mononucleated cells through mitotic slippage. Importantly, analysis of these cells in their following mitosis revealed that a large majority divided in a multipolar manner after assembling multipolar spindles. In these spindles, similarly to binucleated polyploid OVCAR-8 cells, chromosomes formed barriers to spindle pole coalescence (Fig. 8, C and D; and Video 9). We concluded that chromosome barriers in polyploid cells are not necessarily a byproduct of multinucleation.

\section{MT stabilization suppresses multipolarity in polyploid cells} We next investigated whether MT stabilization influenced cell division in polyploid conditions. Motivated by the findings that MT stabilization in simulations (obtained by decreasing their catastrophe rate by $25 \%$ ) showed a clear improvement in centrosome and pole clustering (Fig. 9, A and B; and Video 10), we further tested this possibility in OVCAR-8 cells, using the depletion of the MT-depolymerizing kinesin mitotic centromereassociated kinesin (MCAK). Using small interefering RNAs for $72 \mathrm{~h}$ (siMCAK), MCAK levels were reduced (Fig. S4 C), which led to MT stabilization as shown previously (Desai et al., 1999; Gemble et al., 2019) and an increase in astral MT length easily noticeable in prometaphase cells (Fig. S4, D and E). Strikingly, MT stabilization resulted in a considerable improvement in spindle bipolarity, and the large majority of polyploid cells divided in a bipolar manner (Fig. 9, C and E; and Video 10). Interestingly, this improvement occurred through distinct mechanisms. First, a clear improvement in centrosome clustering was apparent at the beginning of mitosis in $\sim 40.0 \%$ of the cells. In this case, extra centrosomes rapidly clustered in two main poles (Fig. 9, C, E, and F), and the spindle maintained a bipolar status throughout mitosis. Second, in cells where the initial centrosome clustering was not fully efficient and instead of generating two poles, generated three, spindle pole coalescence took place, culminating with bipolar spindle assembly (Fig. 9, D-F). We also analyzed the siMCAK polyploid OVCAR-8 cells that failed to assemble a bipolar spindle (Fig. S4 F). In this case, the chromosome condensation and achievement of a stable chromosome-MT configuration blocked spindle pole coalescence, inhibiting bipolar spindle assembly. These results show that MT stabilization in polyploid cells can favor centrosome clustering and/or spindle pole coalescence generating bipolar spindles. 
A

Polyploid OVCAR-8 cell - Pole coalescence

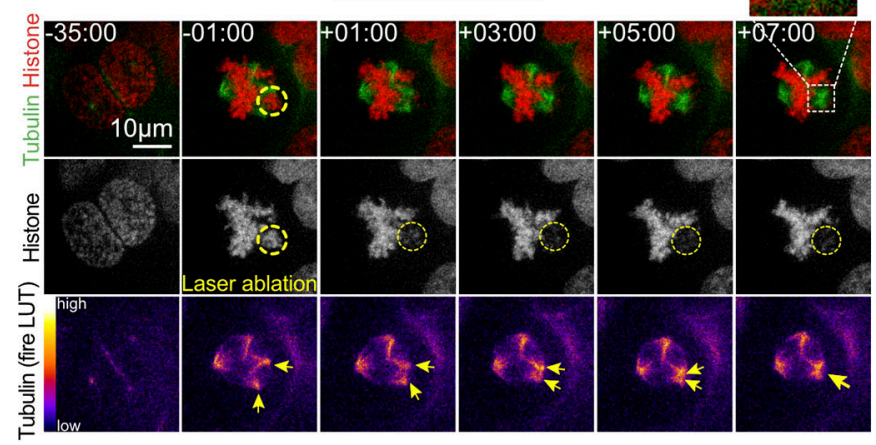

C

Polyploid OVCAR-8 cell - Lack of coalescence + new DNA barrier

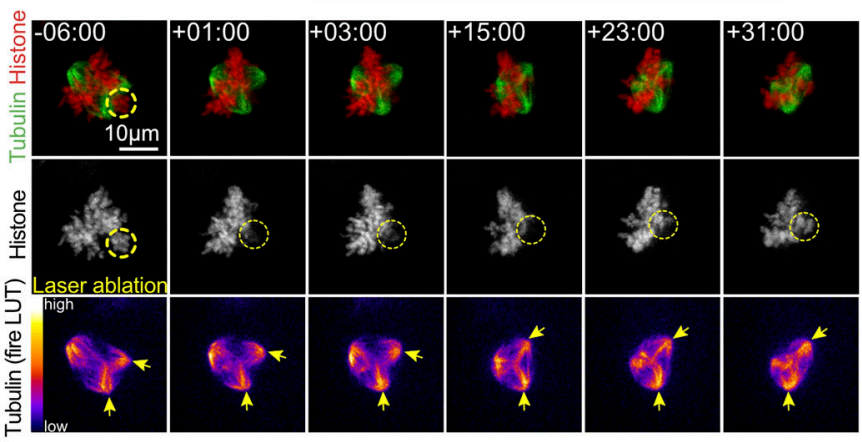

$\mathrm{E}$

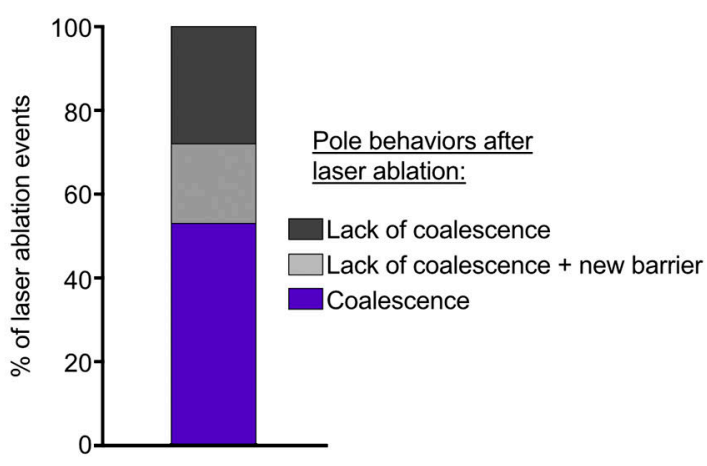

B

Polyploid OVCAR-8 cell - Pole coalescence

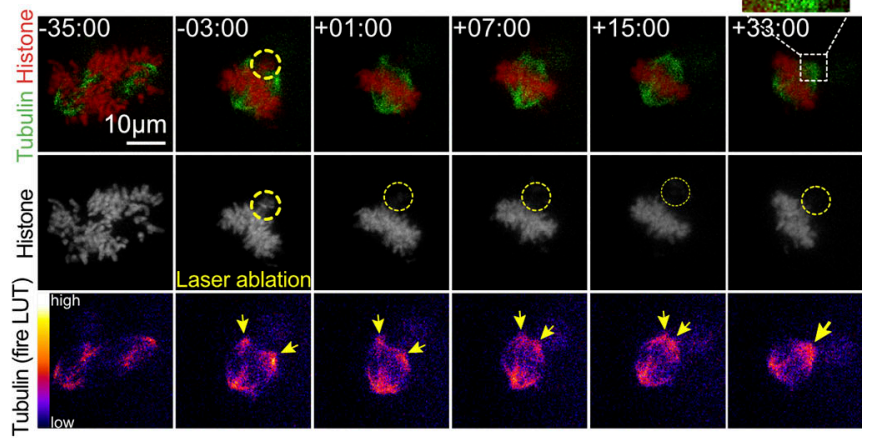

D

Polyploid OVCAR-8 cell - Lack of coalescence

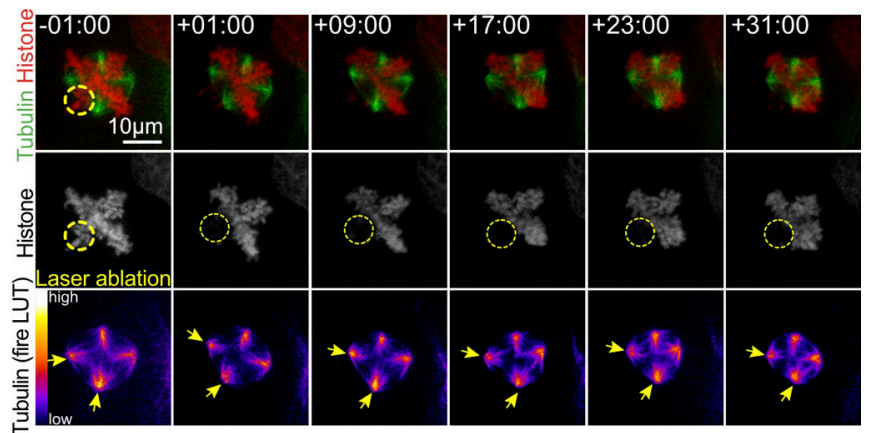

F

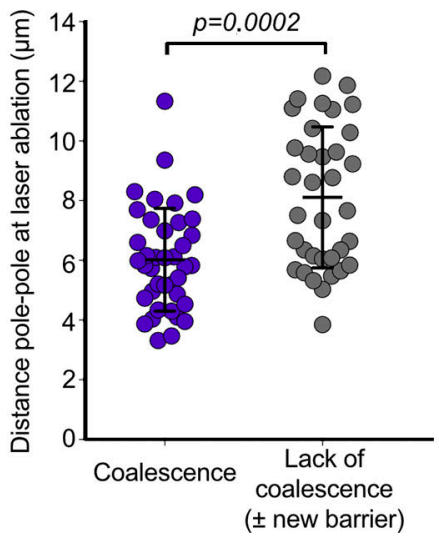

Figure 7. Chromosome laser ablation allows spindle pole coalescence in polyploid OVCAR-8 cells. (A-D) Stills of time-lapse videos of mitotic binucleated polyploid OVCAR-8 cells expressing histone 2B-GFP (red and gray in the middle insets) and incubated with SiR-tubulin (green and fire LUT in the bottom insets). Yellow dotted circles surround the laser ablated area. Time of mitosis is represented in minutes:seconds. Time 00:00 corresponds to the time of laser ablation. (E) Graph bars representing the percentage of laser ablation events leading to the indicated behaviors ( $n=74$ cells from six independent experiments). (F) Dot plot representing the distance between the two poles on both sides of laser ablated DNA in polyploid OVCAR-8 cells ( $n=74$ cells). Statistical significance by a Mann-Whitney test. Error bars represent the mean \pm SD and $p$ the $P$ value.

\section{Discussion}

Here we have analyzed polyploid mitosis in three different model systems: Drosophila NBs, in silico simulations, and the OVCAR-8 cancer cell line. In all cases, an increase in centrosome number, accompanied by increased DNA content, resulted in multipolar spindle assembly and multipolar divisions. We observed that in polyploid mitosis, an initial centrosome clustering step gathers multiple centrosomes in multiple spindle poles. These poles are maintained, separated from each other throughout mitosis, since a second step that should trigger their coalescence into two main poles of a bipolar spindle fails (Fig. 10). Importantly, lack of spindle pole coalescence after 
A

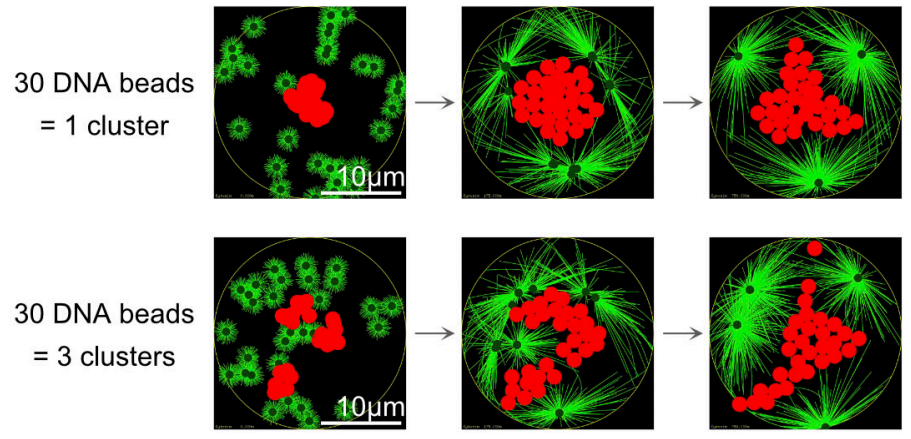

C

Mononucleated polyploid OVCAR-8 cell

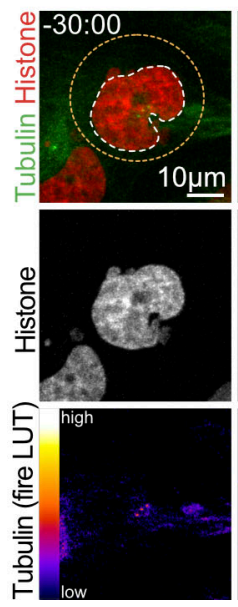

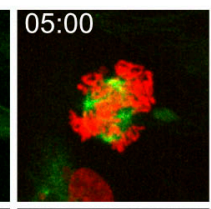
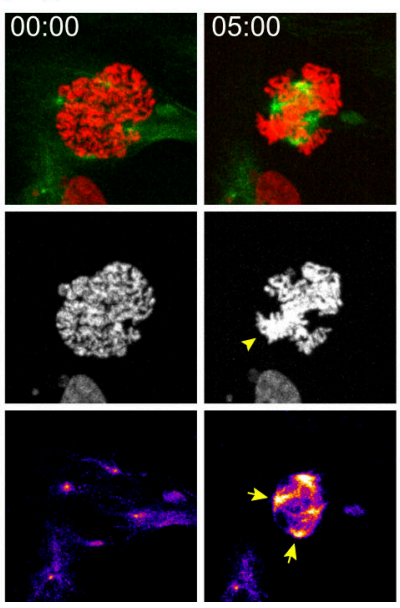
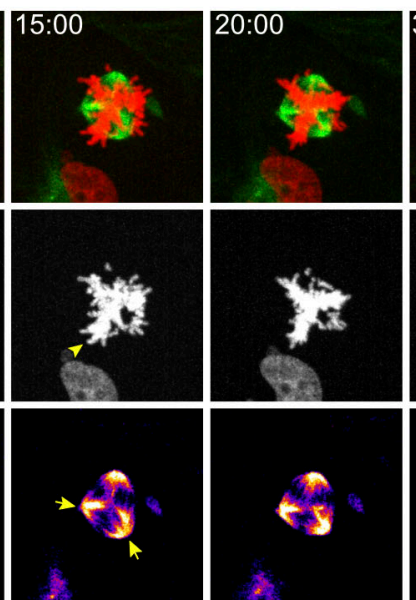
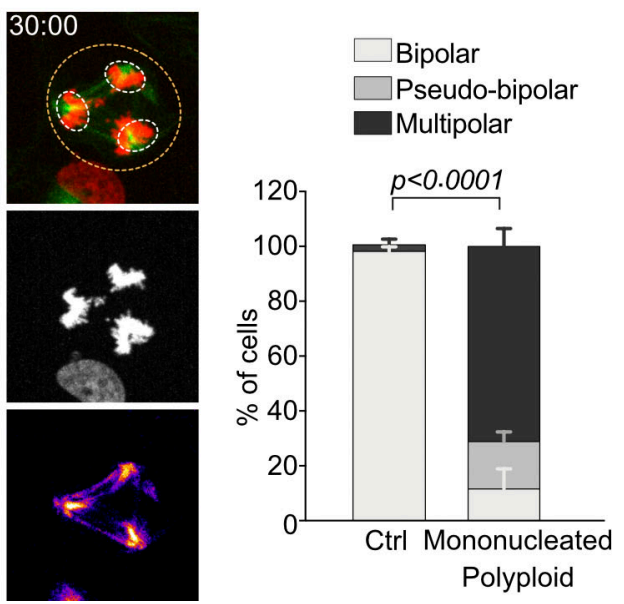

Figure 8. Polyploid OVCAR-8 cells undergo multipolar divisions regardless the number of nuclei at mitotic entry. (A) Representative images of Cytosim-based simulations of spindle formation. (B) Graph bars of the percentage of simulations in each category $(n=30$ simulations/condition). (C) Stills of time-lapse video of mitotic mononucleated polyploid OVCAR-8 cell expressing histone 2B-GFP (red and gray in the middle insets) and incubated with SiRtubulin (green and fire LUT in the bottom insets). Orange and white dotted circles surround cells and nuclei, respectively. Time of mitosis is represented in minutes:seconds. Time 00:00 corresponds to NEBD. (D) Graph bars of the percentage of cells in each category in Ctrl $(n=152$ cells from three independent experiments) and mononucleated polyploid OVCAR- 8 cells ( $n=178$ cells from three independent experiments). Statistical significance by a multiple $t$ test. Error bars represent the mean \pm SD and $p$ the $P$ value between "multipolar" populations.

initial centrosome clustering was also seen in polyploid cells containing a single nucleus. Taking into consideration the fact that bipolar or pseudo-bipolar spindles are assembled in cells that have been manipulated to amplify centrosomes, but not their DNA content (Kwon et al., 2008; Basto et al., 2008; Ganem et al., 2009; Silkworth et al., 2009), our results suggest that extra chromosomes hinder spindle pole coalescence and contribute to the establishment of multipolarity.

It is important to consider that as polyploid cells progress through mitosis and kinetochore-MT attachments become stabilized, abnormal chromosome configurations emerge. It is possible that in this situation, bipolar attachments of chromosomes prevent evolution of the spindle into a bipolar array. In other words, the spatial chromosome configuration achieved through local bipolar attachments using MTs emanating from several MTOCs might generate geometries with stable chromosome barriers that block spindle pole coalescence. Once the SAC has been satisfied, cells can then transit into anaphase in a multipolar manner.

An interesting question raised by this work relates to the equilibrium between chromosome number and bipolar spindle assembly. Eukaryotes, despite extreme differences in genome size, segregate chromosomes using bipolar arrays established, at least during mitosis, by two centrosomes or equivalent organelles. Polyploidization and increased centrosome numbers challenge bipolarity both in cell types that contain small chromosome numbers like in Drosophila (haploid genome, four chromosomes) or human cells (haploid genome, 23 chromosomes, although OVCAR-8 are not diploid cells). Interestingly, we found that even in aneuploid SakOE, mad2 NBs, small chromosome barriers led to multipolarity. In contrast, in OVCAR-8 cells, small chromosome barriers were resolved while large barriers blocked spindle pole coalescence. It is tempting to speculate the existence of a DNA increase threshold that can ultimately influence spindle polarity if extra centrosomes are present.

The CMP and the Augmin pathways did not appear to be major contributors to spindle multipolarity in Drosophila polyploid NBs. Moreover, and in contrast with what has been reported for the large number of MTOCs in mouse oocytes (Watanabe et al., 2016), Augmin did not influence the initial step 


\section{$s_{3} \mathrm{JCB}$}

A

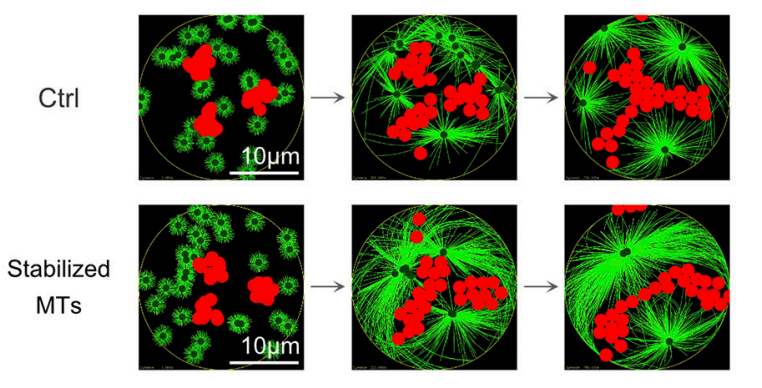

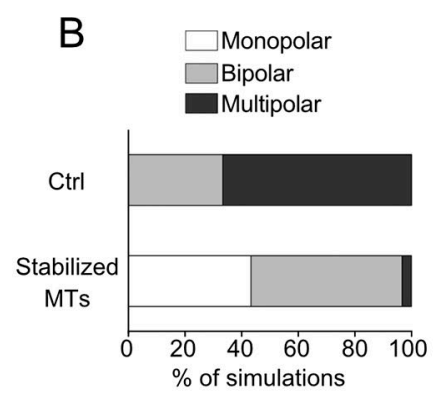

C Polyploid OVCAR-8 cell with stabilized MTs (siMCAK) - Centrosome clustering improvement

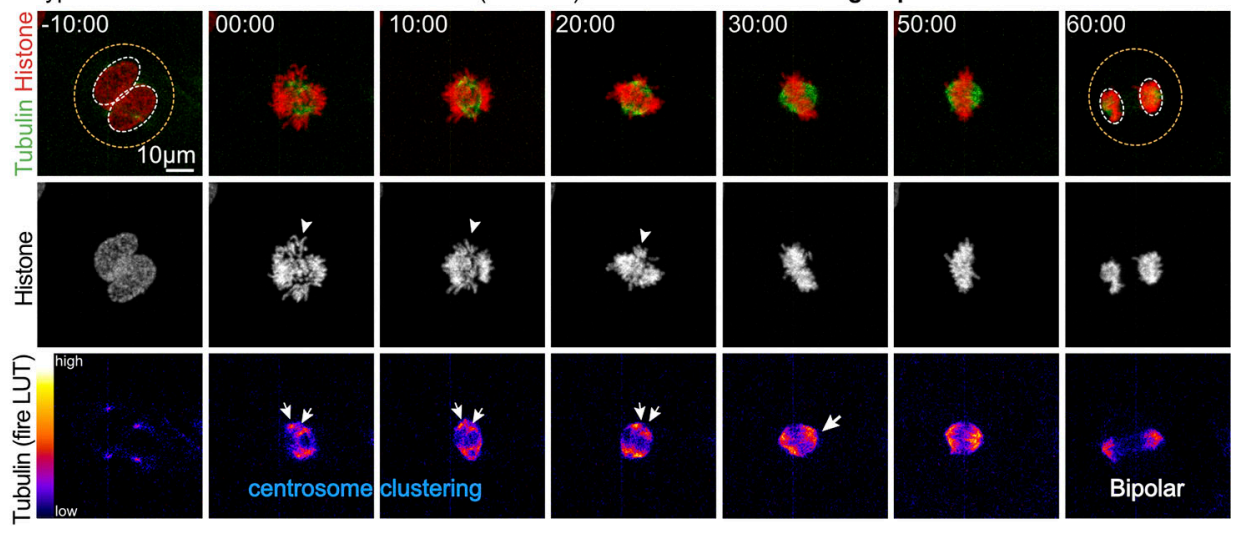

D Polyploid OVCAR-8 cell with stabilized MTs (siMCAK) - Pole coalescence improvement
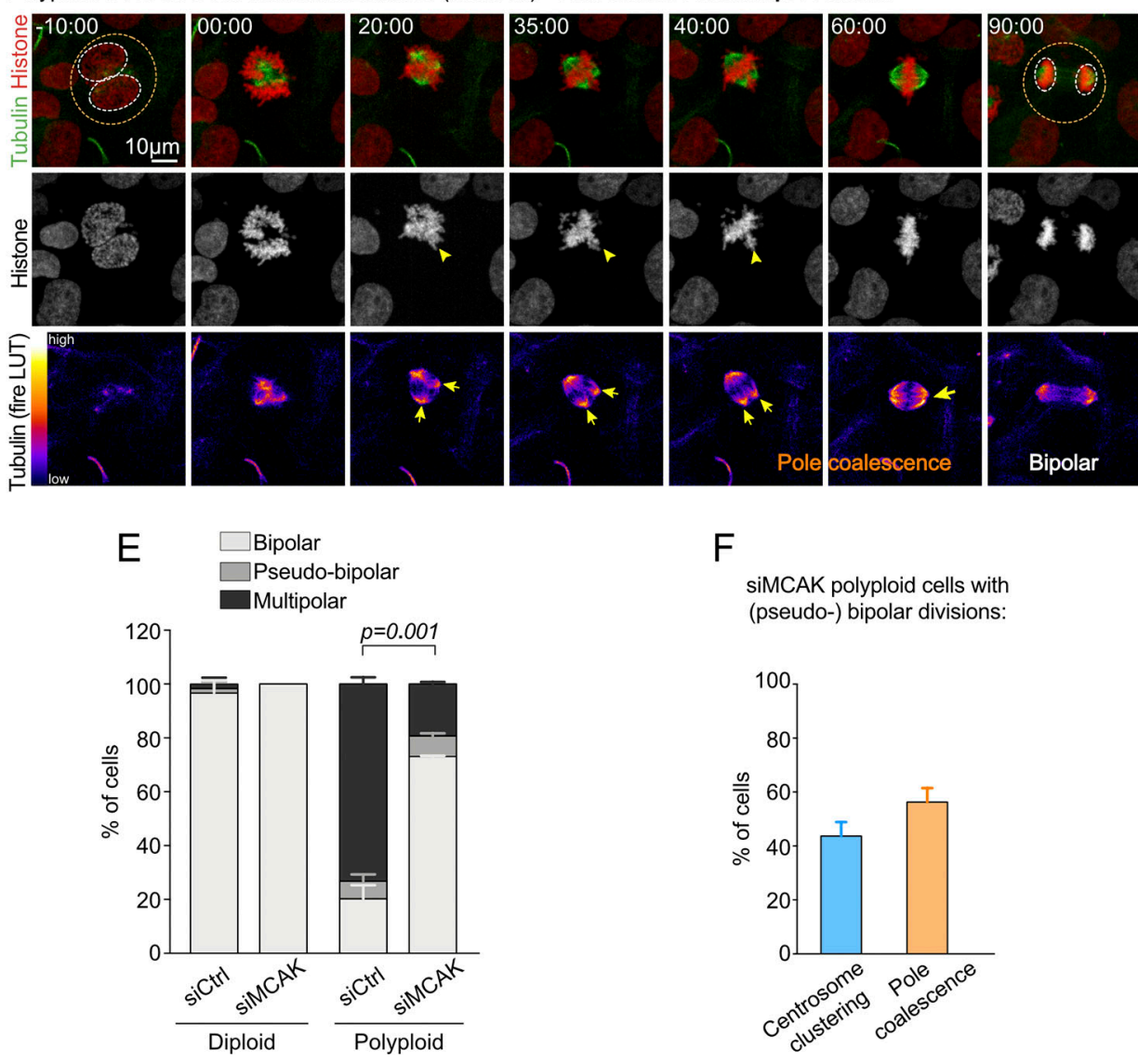
Figure 9. Increased MT stability/length rescues bipolar spindle formation in polyploid OVCAR-8 cells. (A) Representative images of Cytosim-based simulations of spindle formation. (B) Graph bars of the percentage of simulations in each category ( $n=30$ simulations/condition). (C and D) Stills of time-lapse videos of mitotic polyploid OVCAR-8 cells with stabilized MTs (siMCAK) expressing histone 2B-GFP (red and gray in the middle insets) and incubated with SiRtubulin (green and fire LUT in the bottom insets). Orange and white dotted circles surround cells and nuclei, respectively. Time of mitosis is represented in minutes:seconds. Time 00:00 corresponds to NEBD. (E) Graph bars showing the percentage of cells in each category in Ctrl, siCtrl ( $n=96 \mathrm{cells}$ from two independent experiments), Ctrl, siMCAK ( $n=100$ cells from two independent experiments), polyploid, siCtrl ( $n=81$ cells from two independent experiments), and polyploid, siMCAK ( $n=109$ cells from two independent experiments) OVCAR- 8 cells. Statistical significance by a multiple $t$ test. $p$ represents the P value between "multipolar" populations. (F) Graph bars showing the percentage of (pseudo-)bipolar siMCAK, polyploid OVCAR-eight cells presenting an improvement of centrosome clustering or spindle pole coalescence. Error bars represent the mean \pm SD.

of centrosome clustering in Drosophila NBs. Together, these results show cell- and tissue-specific requirements for these pathways in promoting spindle bipolarization. Interestingly, a recent study performed in tetraploid acentriolar mouse oocytes reported the presence of two individual spindles assembled independently around each nucleus (Paim and FitzHarris, 2019), which is not the case in the polyploid cells analyzed here. In contrast to CMP and Augmin, removing centrosomes from small- to medium-sized polyploid NBs generated bipolar spindles that segregated large chromosome masses into two poles. One single bipolar spindle was assembled around polyploid DNA content. However, increased DNA content, typical of large polyploids, did not allow bipolar spindle assembly, revealing an unknown threshold of DNA content (and most likely cell volume or area) permissive to bipolar spindle assembly in the absence of centrosomes. Future work will be required to dissect the limiting factors that condition bipolar spindle assembly in acentriolar polyploid mitosis.
Our work in Drosophila polyploid NBs also confirmed an essential role of the minus-end directed motor Ncd/HSET in the initial centrosome clustering step. However, even if required during early mitosis, the final spindle pole coalescence phase, which is blocked in polyploid cells, does not seem to fail because of limiting Ncd levels, since its overexpression did not lead to any reduction of multipolarity. Surprisingly, however, increased MT stability was sufficient to improve bipolarity. This suggests that longer MTs that promote clustering at mitotic entry and/or spindle pole coalescence later on allow Ncd/HSET to promote bipolarization (Fig. 10).

WGDs are found in a variety of tumors and are associated with CIN and poor tumor prognosis (Bielski et al., 2018; Zack et al., 2013). The tendency to divide multipolarly in polyploid cells does not seem to depend on the number of nuclei at mitotic entry, suggesting that multiple conditions that lead to increased DNA content such as cytokinesis failure, mitotic slippage, and most likely endoreplication can lead to CIN. Here, we show that

Polyploid cell division

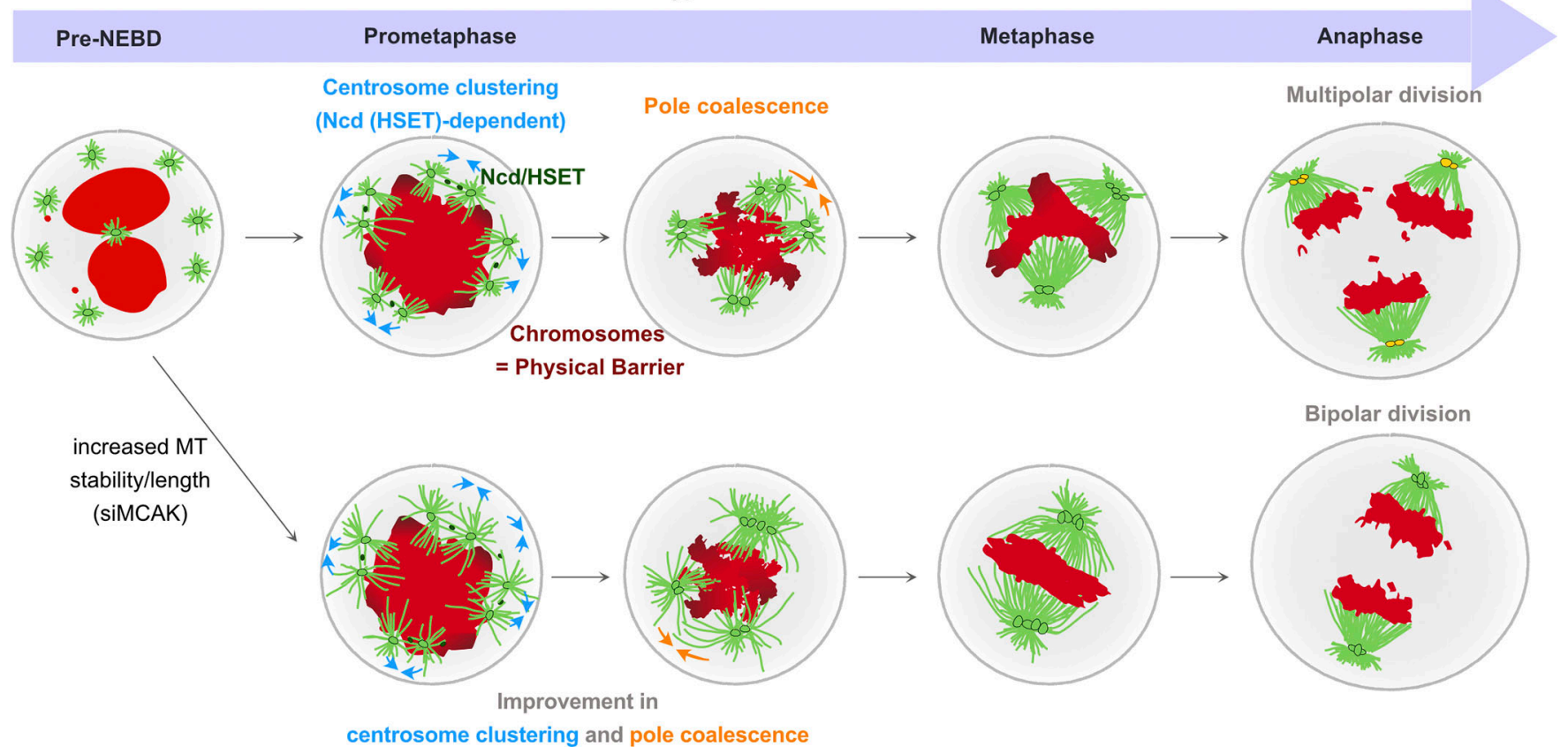

Figure 10. Model of polyploid mitotic division containing multiple centrosomes and nuclei. Top: When a cell with two nuclei (red dots) undergoes NEBD, an Ncd/HSET-dependent clustering step gathers extra centrosomes in multiple active MTOCs. As kinetochore-MT attachments become stabilized, chromosomes can be positioned between two spindle poles and inhibit their coalescence, acting as a physical barrier to bipolarity. At anaphase, chromosomes are segregated into multipolar arrays, giving rise to more than two nuclei. Bottom: In the presence of increased MT stability, centrosomes and spindle poles cluster and coalesce more efficiently, giving rise to bipolar spindles. 
error-prone mitosis in polyploid cells results from the incapacity of these cells to fully coalesce spindle poles containing extra centrosomes due to the presence of chromosome barriers. It is important to take into account the suppression of multipolarity in polyploid cells with increased MT stability. This treatment not only improved centrosome clustering but also permitted spindle pole coalesce into a bipolar configuration. MT polymers, even if stiff, are unlikely to break through condensed mitotic DNA (Ganem and Pellman, 2012; Houchmandzadeh et al., 1997). Thus, the improvement in spindle pole coalescence seems to be explained by increased MT length, allowing the DNA barrier to be surrounded (Fig. 10). Multipolar cell divisions are poorly tolerated, and cancer cells should minimize the generation of unviable daughter cells (Ganem et al., 2009; Kwon et al., 2008). In light of our findings and knowing that WGDs are frequent in cancer, it is possible that clonal expansion of polyploid cancer cells is favored in conditions of increased MT stability.

\section{Materials and methods}

Experimental models

\section{Fly husbandry and fly stocks}

Flies were raised in plastic vials or bottles containing homemade Drosophila culture medium (0.75\% agar, $3.5 \%$ organic wheat flour, $5 \%$ yeast, $5.5 \%$ sugar, $2.5 \%$ nipagin, $1 \%$ penicillin-streptomycin, and $0.4 \%$ propanic acid). Fly stocks were conserved at $18^{\circ} \mathrm{C}$. For temporal activation of RNAi, crosses were kept at $18^{\circ} \mathrm{C}$ and switched to $29^{\circ} \mathrm{C}$ for $24-48 \mathrm{~h}$. Other experimental crosses were maintained at $25^{\circ} \mathrm{C}$. Most fly stocks were obtained from Bloomington Drosophila Stock Center or described in Endow and Komma (1998); Basto et al. (2006); Zhu et al. (2006); and Gogendeau et al.(2015).

\section{Human cell lines}

Cell culture conditions. Cells were maintained at $37^{\circ} \mathrm{C}$ in a $5 \%$ $\mathrm{CO}_{2}$ atmosphere. OVCAR-8 (female) and human embryonic kidney (HEK; female) cells were grown in DMEM F12 containing $10 \%$ fetal bovine serum, $100 \mathrm{U} / \mathrm{ml}$ penicillin, and $100 \mathrm{U} / \mathrm{ml}$ streptomycin.

Cells were routinely checked for mycoplasma and underwent cell authentication by short tandem repeat analysis processed at the Genomics Platform (Department of Translational Research, Institut Curie).

Generation of OVCAR-8 PLK4OE stable cell line. To generate PLK4 inducible stable cell lines from OVCAR- 8 cells, we used a Dox-inducible PLK4 lentiviral expression system (Holland et al., 2012). Viruses were produced in HEK cells, cotransfected with pMD2.G and psPAX2 plasmids using lipofectamine 2000. Viral particles were then used to infect OVCAR- 8 cells for $24 \mathrm{~h}$. Infected cells were selected using bleomycin $(50 \mu \mathrm{g} / \mathrm{ml})$ for $15 \mathrm{~d}$.

Generation of OVCAR-8 H2B-GFP and OVCAR-8 PLK4OE H2B-RFP stable cell lines. To produce viral particles, HEK cells were transfected with psPAX2, pMD2.G, and pHR_dSV40-H2B-GFP or pHR_dSV40-H2B-RFP plasmids using FuGENE HD Transfection Reagent or PEI MAX in OptiMEM medium, respectively. Cells were incubated at $37^{\circ} \mathrm{C}$ in a $5 \% \mathrm{CO}_{2}$ atmosphere for $16 \mathrm{~h}$. The growth media were removed and replaced by $5 \mathrm{ml}$ fresh
OptiMEM. The day after, viral particles were isolated by filtering the medium containing the viral particles through a $0.45-\mu \mathrm{m}$ filter. Then, OVCAR- 8 or OVCAR- 8 PLK4OE cells were incubated with viral particles in the presence of polybrene $8 \mu \mathrm{g} / \mathrm{ml}$ for 24 h. OVCAR-8 GFP-positive and OVCAR-8 PLK4OE RFP-positive cells were then collected using BD FACS Aria IIIu (BD FACSDiva Software Version 8.0.1). To preserve inherent OVCAR-8 heterogeneity, a bulk population of GFP-positive and RFP-positive cells has been sorted.

\section{Drug treatments for human cell lines}

To induce centrosome amplification, OVCAR-8 PLK4OE H2BRFP cells were plated at the appropriate confluence and treated for $96 \mathrm{~h}$ with Dox $(1 \mu \mathrm{g} / \mathrm{ml})$ or DMSO (drug diluent $0.0001 \%$ ) for control before immunofluorescence and live imaging.

OVCAR-8 H2B-GFP cells were plated $24 \mathrm{~h}$ before drug treatment at the appropriate confluence. To induce cytokinesis failure, cells were treated for $1 \mathrm{~h}$ at $37^{\circ} \mathrm{C}$ with latrunculin $\mathrm{B}$ (5 $\mu \mathrm{M})$ or ethanol (drug diluent, $0.015 \%)$ for control and then washed in PBS. The efficiency of the drug treatment was assessed by cell rounding and was considered as time 0 . To induce mitotic slippage, cells were treated overnight $(\mathrm{O} / \mathrm{N})$ with the combination of MPI-0479605 (MPS1 inhibitor, $0.5 \mu \mathrm{M}$ ) and monastrol (Eg5 inhibitor, $50 \mu \mathrm{M}$ ) or DMSO (drug diluent $0.15 \%$ ) for control. Drugs were washed out $3 \times$ in PBS. Immunofluorescence and live imaging were performed $24 \mathrm{~h}$ or $12 \mathrm{~h}$ after drugs washout in proliferative medium. $4 \mathrm{~h}$ before live imaging, cells were treated with SiR-tubulin (33 $\mathrm{nM})$ and Verapamil (1 nM).

\section{MCAK depletion in human cell lines}

To deplete MCAK, OVCAR-8 H2B-GFP cells were plated at the appropriate confluence and treated for $72 \mathrm{~h}$ with $10 \mathrm{nM}$ of siMCAK or control siRNAs (siCtrl). siRNA mixes were prepared using the jetPRIME kit and following the manufacturer's protocol. Reagents and materials are listed in Table S3.

\section{Methods and microscope image acquisition Live imaging of Drosophila larval brains}

Mid third-instar larval (L3) brains were dissected in Schneider's Drosophila medium supplemented with $10 \%$ heat-inactivated fetal bovine serum, penicillin $(100 \mathrm{U} / \mathrm{ml})$, and streptomycin $(100 \mu \mathrm{g} / \mathrm{ml})$. Several brains were placed on a glass-bottom dish with near $10 \mu \mathrm{l}$ of medium, covered with a permeable membrane, and sealed around the membrane borders with oil $10 \mathrm{~S}$ Voltalef. Images were acquired with $60 \times$ oil objective (NA 1.4) on two microscopes: an Inverted Spinning Disk Confocal Roper/ Nikon (a Yokagawa CSU-X1 spinning head mounted on a Nikon Ti-E inverted microscope equipped with a camera EMCCD $512 \times$ 512 Evolve; Photometrics) and the wide-field Inverted Spinning Disk Confocal Gattaca/Nikon (a Yokagawa CSU-W1 spinning head mounted on a Nikon Ti-E inverted microscope equipped with a camera complementary metal-oxide semiconductor 1,200 × 1,200 Prime95B; Photometrics), controlled by Metamorph software. For both microscopes, images were acquired at time intervals spanning from $30 \mathrm{~s}$ (diploid conditions) to $2 \mathrm{~min}$ (polyploid conditions) and 30 to $50 \mathrm{Z}$-stacks of 1-1.5 $\mu \mathrm{m}$. 


\section{Immunofluorescence of Drosophila larval squashed brains}

L3 brains were dissected in PBS and fixed for 20-40 min at RT in $4 \%$ formaldehyde diluted in PBS. After fixation, brains were transferred in a drop of $45 \%$ acetic acid on a coverslip for $15 \mathrm{~s}$ and then immediately moved in a drop of $60 \%$ acetic acid. Acetic acid was diluted in water. A slide was then placed on top of the coverslip, and brains were squashed with a pencil until the tissue appeared transparent. The slide was rapidly flash frozen in liquid nitrogen before the removal of the coverslip using a sharp blade. Slides were incubated $7 \mathrm{~min}$ in $-20^{\circ} \mathrm{C}$ methanol, washed, and permeabilized three times for $10 \mathrm{~min}$ in PBST $0.1 \%$ solution (PBS, $0.1 \%$ Triton X-100, and $0.02 \%$ sodium azide) at RT. Slides were dried at RT before incubation with $10 \mu \mathrm{l}$ of primary antibodies diluted in PBST $0.1 \%$ and covered with a coverslip in a humid chamber at $4^{\circ} \mathrm{C} \mathrm{O} / \mathrm{N}$. After removal of the coverslips, slides were washed $3 \mathrm{x}$ for $10 \mathrm{~min}$ in PBST $0.1 \%$ at RT. $10 \mu \mathrm{l}$ of secondary antibody dilution was added to the slides, and slides were covered with coverslips in a dark, humid chamber and incubated $3 \mathrm{~h}$ at $25^{\circ} \mathrm{C}$. Slides were washed $3 \mathrm{x}$ for $10 \mathrm{~min}$ in PBST $0.1 \%$ and incubated in $0.5 \mu \mathrm{g} / \mathrm{ml}$ Hoechst for $15 \mathrm{~min}$ at RT. Slides were mounted using a $22 \times 22 \mathrm{~mm}$ coverslip using $10 \mu \mathrm{l}$ of mounting medium (1.25\% n-propyl gallate, $75 \%$ glycerol, $25 \%$ $\mathrm{H}_{2} \mathrm{O}$ ). Images were acquired with $40 \times$ (NA 1.25), 63× (NA 1.32), or 100× (NA 1.4) oil objectives on an Epifluorescent Upright microscope Leica DM6000 equipped with a camera CCD, 1,392 $\times$ 1,040 CoolSnap HQ (Photometrics). Intervals for Z-stack acquisitions were set to 0.3 to $1 \mu \mathrm{m}$ using Metamorph software.

\section{Immunofluorescence of Drosophila larval whole mount brains} L3 brains were dissected in PBS and fixed for $30 \mathrm{~min}$ at RT in $4 \%$ paraformaldehyde diluted in PBS. After fixation, brains were washed and permeabilized three times for $10 \mathrm{~min}$ in PBST $0.3 \%$ (PBS, $0.3 \%$ Triton $\mathrm{X}-100$, and $0.02 \%$ sodium azide). Brains were then incubated in primary antibodies diluted in PBST $0.3 \%$, at $4^{\circ} \mathrm{C} \mathrm{O} / \mathrm{N}$ in a humid chamber. After $3 \times 10$ min washes in PBST $0.3 \%$, brains were incubated in secondary antibodies and DAPI dilution (in PBST 0.3\%), either for several hours at $25^{\circ} \mathrm{C}$ or $\mathrm{O} / \mathrm{N}$ at $4^{\circ} \mathrm{C}$, protected from light in a humid chamber. After three washes for $10 \mathrm{~min}$ in PBST $0.3 \%$, brains were rinsed in PBS and mounted using 12-mm coverslips with $5 \mu \mathrm{l}$ of mounting medium (1.25\% n-propyl gallate, $75 \%$ glycerol, $25 \% \mathrm{H}_{2} \mathrm{O}$ ). Images were acquired on a confocal Nikon A1R Ti-E inverted microscope with a $60 \times$ (NA 1.4) oil objective. The interval for Z-stack acquisitions was set up with $0.3 \mu \mathrm{m}$ using the NIS Element software.

\section{Immunofluorescent staining of human cells}

Cells were plated on coverslips in 12-well plates and treated with the indicated drugs. The day of the immunofluorescence, cells were fixed using cold methanol $\left(7 \mathrm{~min}\right.$ at $\left.-20^{\circ} \mathrm{C}\right)$. Cells were washed three times using PBST (PBS $1 \times+0.1 \%$ Triton $\mathrm{X}-100+$ $0.02 \%$ sodium azide) and incubated with PBST + BSA $0.5 \%$ for 30 min at RT. After three washes with PBST, primary and secondary antibodies (1:250 concentration) were incubated in PBST + BSA $0.5 \%$ for $1 \mathrm{~h}$ and $30 \mathrm{~min}$ at RT, respectively. Nuclear DNA was detected by incubating with DAPI diluted in PBS, and slides were mounted with mounting medium (1.25\% n-propyl gallate, $75 \%$ glycerol, and $25 \% \mathrm{H}_{2} \mathrm{O}$ ). Images were acquired on an upright widefield microscope (DM6B, Leica Systems) equipped with a motorized XY and a $40 \times$ objective (HCX PL APO 100 × /1.40-0.70 Oil from Leica). Acquisitions were performed using Metamorph software and a sCMOS camera (Flash 4V2, Hamamatsu). Stacks of conventional fluorescence images were collected automatically at a Z-distance of $0.2 \mu \mathrm{m}$ (Metamorph software). Images are presented as maximum intensity projections generated with ImageJ software, from stacks deconvolved with an extension of Metamorph software.

\section{Live imaging of human cells and laser ablation}

Cells were plated on a dish and treated with the indicated drug. For live imaging, images were acquired on a spinning disk microscope (Gataca Systems). Based on a CSU-W1 (Yokogawa), the spinning head was mounted on an inverted Eclipse Ti2 microscope equipped with a motorized XY Stage (Nikon). Images were acquired through a $40 \times 1.4$ NA Plan-Apo objective, with a sCMOS camera (Prime95B, Photometrics). Optical sectioning was achieved using a piezo stage (Nano-z series, Mad City Lab). Gataca Systems' laser bench was equipped with 405-, 491-, and 561-nm laser diodes, delivering $150 \mathrm{~mW}$ each, coupled to the spinning disk head through a single mode fiber. Multidimensional acquisitions were performed using Metamorph 7.10.1 software. Stacks of conventional fluorescence images were collected automatically at a Z-distance of $1.5 \mu \mathrm{m}$ (Metamorph software). Images are presented as maximum intensity projections generated with ImageJ software, from stacks deconvolved with an extension of Metamorph software. For laser ablation experiments, images were acquired on an Inverted Eclipse Ti-E (Nikon) + Spinning disk CSU-X1 (Yokogawa) integrated in Metamorph software by Gataca Systems. Images were acquired through a 100× 1.3 NA Plan Fluor (UV) objective with a sCMOS camera (Prie BSI, Photometrics). Optical sectioning was achieved using a piezo stage (Nano-z series, Mad City Lab). Gataca Systems' laser bench was equipped with 405-, 488-, 561-, and 633-nm laser diodes, delivering $100 \mathrm{~mW}, 70 \mathrm{~mW}, 60 \mathrm{~mW}$, and $35 \mathrm{~mW}$, respectively. Laser ablation was performed with a Photoablation module ILAS2 (Gataca Systems) equipped with a pulsed laser at $355 \mathrm{nM}$ for nanoablation. Laser ablation was performed with spot length of $100 \mathrm{~nm}$ of UV laser, between two time points of acquisition.

\section{Western blot siMCAK OVCAR-8 cells}

Cells were lysed in the lysis buffer composed of $8 \mathrm{M}$ urea, $50 \mathrm{mM}$ Tris $\mathrm{HCl}, \mathrm{pH} 7.5$, and $150 \mathrm{mM} \beta$-mercaptoethanol, sonicated and heated at $95^{\circ} \mathrm{C}$ for $10 \mathrm{~min}$. Samples were subjected to electrophoresis in NuPAGE Novex 4-12\% Bis-Tris precast gels. Protein fractions from the gel were electrophoretically transferred to polyvinylidene fluoride membranes. After $1 \mathrm{~h}$ saturation in PBS containing $5 \%$ dry nonfat milk and $0.5 \%$ Tween 20 , the membranes were incubated with primary antibody against MCAK or a-tubulin (for control) diluted in PBS containing 5\% dry nonfat milk and $0.5 \%$ Tween 20 for $1 \mathrm{~h}$. After three 10 -min washes with PBS 0.5\% Tween 20, membranes were incubated for $45 \mathrm{~min}$ with peroxidase-conjugated antibodies. Membranes were then washed three times with PBS containing $0.5 \%$ Tween 20 , and the reaction was developed according to the manufacturer's specifications using ECL reagent. 


\section{Computer simulations}

Simulations were performed using Cytosim considering mitotic cells as spherical entities (Letort et al., 2016). We confined the MTs and motors in a circular shape (2D). Centrosomes were modeled as asters nucleating MTs radially. They were initially randomly placed inside the cell, corresponding to the experimental situations just after NEBD. MTs were considered as flexible polymers with static minus ends and dynamic plus ends that could grow and shrink according to the dynamic instability model (Gittes et al., 1993; Dogterom and Yurke, 1997; Gallaud et al., 2014). The kinesin motors Ncd were represented as entities composed of two separate MT-binding "heads," one static (corresponding to the nonmotor domain) and one that could move toward the MT minus ends (Howard et al., 1989; Oladipo et al., 2007; Rupp and Nédélec, 2012; Letort et al., 2019). Ncd was shown to bind and slide anti-parallel MTs and cross-link parallel MTs (Fink et al., 2009). Here, as we were interested in the clustering effect (which depends on anti-parallel sliding), we constrained Ncd-like entities to bind exclusively to anti-parallel MTs. This reduces the amount of entities to simulate. Finally, we used two different representations of the chromatin: (1) the metaphase plate was simulated as a static obstacle (an area of a defined shape) that MTs cannot cross nor deform based on Houchmandzadeh et al. (1997), and (2) the chromatin was simulated as mobile beads (without intrinsic motion) that could be pushed by the MTs (Letort et al., 2019). In condition 1, MTs and the initial position of the centrosomes were excluded from this area. In condition 2, the DNA beads were initially placed in two to five groups (mimicking the nuclei number at NEBD) of 0-50 DNA beads each, randomly distributed within a disk of radius slightly smaller than the cell radius (so that the cluster will not be blocked against the cortex). In both conditions, we did not consider MT nucleation from the plate/beads to simplify the model. MTs were prevented from sliding on the surface of the plate/beads by the presence of plus-end tracking entities (mimicking chromokinesins present on chromosomes or capture by prometaphase kinetochores) similar to simulations from Lacroix et al., (2018). Parameters in the simulations were chosen to match the experimental conditions when measurable or from the literature when available. Note that values of these parameters were adjusted to check that the numerical conclusions were not greatly affected by this choice. All parameters are detailed in Table S2.

To further test our interpretation of DNA acting as a physical barrier to centrosome clustering, we took advantage of the flexibility of the simulations and explored numerically different scenarios, changing only one parameter at a time. In our previous simulations (condition 2), we kept a constant number of 30 centrosomes and varied the DNA content, from 9 to 41 DNA beads distributed in two to five initial groups (approximate nuclei at NEBD). Conversely, we varied the number of centrosome $(10,30$, or 50 centrosomes), keeping a constant but high DNA content (30 beads of DNA, initially distributed in three randomly placed clusters). Then we tested the effect of the initial geometry of the DNA by varying the number of initial beads group ( 1 or 3 ), keeping a constant number of beads (30) and centrosomes (30). Finally, the stability of MTs was increased by decreasing by $25 \%$ their catastrophe rate in our default condition (30 centrosomes, 30 beads of DNA in three initial clusters), in order to increase their length.

\section{Image quantification and statistical analysis}

Image analysis and quantifications were performed using Fiji software. Centrosomes were manually counted and identified as the colocalization of Cnn (Lucas and Raff, 2007) and Plp (Martinez-Campos et al., 2004) markers on Drosophila whole mount brains. Centrioles were manually counted and identified as the colocalization of Centrin 3 dots and CEP192 (VargasHurtado et al., 2019) for OVCAR-8 cells delimitated with the membrane marker $\mathrm{N}$-cadherin. The bipolar/multipolar state of the mitotic spindle, the number of nuclei, the cell and DNA area, and the mitotic timing were assessed on live-imaging data. For all Drosophila polyploid NBs, quantifications were done on cells with an area inferior to $6,000 \mu \mathrm{m}^{2}$ to be able to compare all genetic conditions. To calculate the nuclear index (number of nuclei at mitotic exit over mitotic entry relative to the number of daughter cells), the number of nuclei was manually counted for all Z-stacks at preNEBD and at anaphase, when nuclei are individualized, before chromosome decondensation. This ratio was then normalized by the number of cells generated after anaphase, two in diploid conditions and one in polyploid conditions (cytokinesis failure). Knowing the time intervals between acquired frames, the mitotic timing was calculated from NEBD to anaphase onset. The cell or DNA area was calculated using the "Freehand selection" tool in Fiji software to draw cell or DNA contour of Drosophila NBs in metaphase. In polyploid LatB-treated OVCAR-8 cells that presented four poles in prometaphase, the four chromosome barriers were measured using the "Freehand selection" tool. We analyzed cells that then evolve into a tripolar spindle configuration.

In siCtrl/siMCAK polyploid OVCAR- 8 cells, the length of astral MTs was manually measured using the "Freehand selection" tool in Fiji software. We measured the two to four longest MTs for each pole of eight to eleven prometaphase cells.

All statistical analyses were performed in Prism software (see figure legends for details). For all the analysis of the correlation between the number of nuclei at anaphase or mitotic timing or DNA area and NB area, the correlation coefficient $r$ measures the strength of the relationship between the two variables, while the regression slope represents the rate of change. In other words, the slope indicates to what extent the number of nuclei increases with cell area. For the figures, images were processed on Photoshop and Affinity Photo, and mounted using Illustrator and Affinity Designer.

\section{Fly genetic crosses}

Crosses for Fig. 1

Fixed analysis. Control: $\mathrm{wt}[118]$. Line maintained at $25^{\circ} \mathrm{C}$. Polyploid: Pav ${ }^{\text {RNAi}} /$ Cyo-GFP $x$ Act-GAL4-GAL80 ${ }^{\text {ts }} /$ Cyo-GFP. Crosses were maintained at $18^{\circ} \mathrm{C}$ after egg laying until reaching first/second instar stages and then switched to $29^{\circ} \mathrm{C}$ for $24-48 \mathrm{~h}$.

Live imaging. Control: Tub-GFP, Hist-RFP/Cyo-GFP; ActinGAL4-GAL80 ${ }^{\text {ts }} / \mathrm{TM} 6, \mathrm{~Tb}$. . Pav ${ }^{\mathrm{KD}}: \mathrm{Pav}^{\mathrm{RNAi}} /$ Cyo-GFP $\mathrm{x}$ Tub-GFP, 
Hist-RFP/Cyo-GFP; Act-GAL4-GAL80"s/TM6,Tb. Crosses were maintained at $18^{\circ} \mathrm{C}$ after egg laying until reaching first/second instar stages and then switched to $29^{\circ} \mathrm{C}$ for $24-48 \mathrm{~h}$.

\section{Crosses for Fig. 3}

Live imaging. Polyploid, sas4 $4^{\text {mut }} \mathrm{Pav}^{\mathrm{RNAi}}$,Hist-RFP/Cyo-GFP;

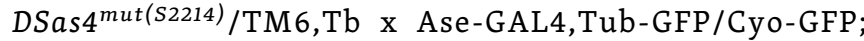

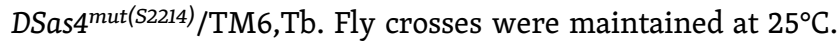

\section{Crosses for Fig. 4}

Fixed analysis. Control: wt[118]. Line maintained at $25^{\circ} \mathrm{C}$. Polyploid: $\mathrm{Pav}^{\mathrm{RNAi}} / \mathrm{Cyo-GFP} \mathrm{x}$ Act-GAL4-GAL80 ${ }^{\mathrm{ts}} / \mathrm{Cyo}-\mathrm{GFP}$. Crosses were maintained at $18^{\circ} \mathrm{C}$ after egg laying until reaching first/second instar stages and then switched to $29^{\circ} \mathrm{C}$ for $24-48 \mathrm{~h}$.

Live imaging. Diploid: Spd2-GFP,Hist-GFP/Cyo-GFP. Polyploid: $\mathrm{Pav}^{\mathrm{RNAi}} / \mathrm{Cyo}-\mathrm{GFP} \mathrm{x}$ Spd2-GFP,Hist-RFP/Cyo-GFP; ActGAL4-GAL80 ${ }^{\text {ts }} / \mathrm{TM} 6, \mathrm{~Tb}$. Crosses were maintained at $18^{\circ} \mathrm{C}$ after egg laying until reaching first/second instar stages and then switched to $29^{\circ} \mathrm{C}$ for $24-48 \mathrm{~h}$.

\section{Crosses for Fig. S1}

Live imaging. Diploid, sas4 $4^{m u t}$ : Tub-GFP,Hist-RFP/Cyo-GFP; DSas4 $^{\text {mut(S2214)/TM6,Tb.. Diploid, Mars }}{ }^{\mathrm{KD}}$ : Tub-GFP,Hist-RFP/ Cyo-GFP; mars ${ }^{\mathrm{RNAi}} / \mathrm{TM} 6, \mathrm{~Tb}$ x Act-GAL4-GAL80 ${ }^{\text {ts }} / \mathrm{TM} 6, \mathrm{~Tb}$. Polyploid, Mars ${ }^{\mathrm{KD}}$ : Pav ${ }^{\mathrm{RNAi}} / \mathrm{Cyo}-\mathrm{GFP} ;$ mars $^{\mathrm{RNAi}} / \mathrm{TM} 6, \mathrm{~Tb} \times \mathrm{Tub}$ GFP,Hist-RFP/Cyo-GFP; Act-GAL4-GAL80 ${ }^{\text {ts }} / \mathrm{TM6,Tb.} \mathrm{Diploid,}$ Aug ${ }^{\mathrm{KD}}$ : Tub-GFP,Hist-RFP/Cyo-GFP; dgt2RNAi/TM6,Tb x ActGAL4-GAL80 5 /TM6, Tb. Polyploid, AugKD: Pav RNAi/Cyo-GFP; dgt2 ${ }^{\text {RNAi}} / \mathrm{TM} 6, \mathrm{~Tb} \times \mathrm{x}$ Tub-GFP,Hist-RFP/Cyo-GFP; Act-GAL4GAL80 ${ }^{\text {ts }} / \mathrm{TM} 6, \mathrm{~Tb}$. Crosses were maintained at $18^{\circ} \mathrm{C}$ after egg laying and until reaching first/second instar stages and then switched to $29^{\circ} \mathrm{C}$ for $24-48 \mathrm{~h}$.

\section{Crosses for Fig. S2}

Live imaging. Diploid, ncd ${ }^{m u t}$ : Tub-GFP,Hist-RFP/Cyo-GFP; $n c d^{1} / \mathrm{TM} 6, \mathrm{~Tb}$. Polyploid, ncd ${ }^{m u t}$ : Pav ${ }^{\mathrm{RNAi}}$, Hist-RFP/Cyo-GFP; $n c d^{1} / \mathrm{TM} 6, \mathrm{~Tb}$ x Asense-GAL4,Tub-GFP/Cyo-GFP; $n c d^{1} / \mathrm{TM} 6, \mathrm{~Tb}$. Diploid, Ncd-GFP: Ncd-GFP/Cyo-GFP; Ncd-GFP, $n c d^{1}, c a^{n d} /$ TM6,Tb. Crosses were maintained at $25^{\circ} \mathrm{C}$.. Polyploid Ncd-GFP: $\mathrm{Pav}^{\mathrm{RNAi}}$, Hist-RFP/Cyo-GFP; Ncd-GFP, $n c d^{1}, c^{\text {nd }} / \mathrm{TM} 6, \mathrm{~Tb} \times \mathrm{Ncd}-$ GFP/Cyo-GFP; Act-GAL4-GAL80 ${ }^{\text {ts }} / \mathrm{TM6}$, Tb. Crosses were maintained at $18^{\circ} \mathrm{C}$ after egg laying until reaching first/second instar stages and then switched to $29^{\circ} \mathrm{C}$ for $24-48 \mathrm{~h}$.

\section{Crosses for Fig. $\mathrm{S3}$}

Live imaging. SakOE, mad2 ${ }^{\text {mut }}$ : pUbqSak-\#68, mad2 ${ }^{\text {mut }} / \mathrm{TM} 6, \mathrm{~Tb}$. $\mathrm{X}$ Tub-GFP,Hist-RFP/Cyo-GFP; mad2 ${ }^{\text {mut }} / \mathrm{TM} 6, \mathrm{~Tb}$. Crosses were maintained at $25^{\circ} \mathrm{C}$.

\section{Online supplemental material}

Fig. S1 shows that diploid and polyploid NBs depleted for MTnucleating pathways undergo bipolar and multipolar divisions. Fig. S2 shows that centrosome clustering takes place in polyploid mitosis, but it is not sufficient to promote bipolar spindle assembly. Fig. S3 shows that chromosomes as physical barriers can perturb centrosome clustering in NBs with centrosome amplification. Fig. S4 shows that PLK4OE and MCAK depletion, respectively, induce centrosome amplification and increase astral MT length. Table S1 summarizes all mitotic parameters calculated in the different diploid and polyploid conditions. Table S2 and Table S3 present the lists of computer simulations parameters and of materials and reagents, respectively. Videos $1,2,3$, and 4 show mitotic divisions in diploid, small, and large polyploid NBs (Video 1), in diploid, sas4 ${ }^{m u t}$, small and large polyploid, sas4 ${ }^{m u t} \mathrm{NBs}$ (Video 2), in diploid Mars ${ }^{\mathrm{KD}}$, diploid Aug $^{\mathrm{KD}}$, diploid ncd ${ }^{m u t}$, and diploid NcdOE NBs (Video 3), or in polyploid Mars ${ }^{\mathrm{KD}}$, polyploid Aug ${ }^{\mathrm{KD}}$, polyploid $n \mathrm{~d}^{m u t}$, and polyploid NcdOE NBs (Video 4). Video 5 shows centrosome clustering in polyploid NBs. Video 6 shows computer simulations of spindle assembly according to centrosome number, DNA content, and DNA shape. Video 7 shows Ctrl, PLK4OE, and polyploid OVCAR- 8 cells mitotic division. Video 8 shows experiments of laser ablation in polyploid OVCAR- 8 cells. Videos 9 and 10 show experimental and simulated mitotic divisions in mononucleated polyploid cells (Video 9) or polyploid cells with increased MT stability (Video 10).

\section{Acknowledgments}

We thank the Plateforme d'Imagerie Cellulaire et Tissulaire (PiCT)-Infrastructure en Biologie Santé et Agronomie (IBiSA) platform and Nikon Imaging Center at Institut Curie for microscopy and V. Fraisier for outstanding help with image acquisition and advice. We thank V. Marthiens, D. Vargas-Hurtado, D. Fachinetti, and S. Passemard for helpful discussion and/or comments on the manuscript. We thank A. Holland (Johns Hopkins School of Medicine, Baltimore, MD) for providing the PLK4 plasmid.

This work was supported by a European Research Council consolidator grant (ChromoNumber LS3, ERC-2016-COG), Institut Curie, and Centre National de la Recherche Scientifique. M. Nano was funded by La Ligue Nationale Contre le Cancer and Fondation pour la Recherche Medicale (FRM; FDT20160435352) grants and A. Goupil by an FRM (ECO20170637529) fellowship. G. Letort was funded by ANR (Agence Nationale de la Recherche,ANR-16-CE13 to M.-E. Terret, College de France, Paris, France). The Basto laboratory is a member of the Labex CelTisPhyBio (ANR-11-LABX-0038 and ANR-10-IDEX-0001-02).

The authors declare no competing financial interests.

Author contributions: A. Goupil performed the large majority of the experiments, analyzed the data, and generated the figures and videos. $M$. Nano conceived the project, performed the initial experiments, and established the majority of Drosophila lines and tools. G. Letort generated the simulation models and contributed to the discussions. S. Gemble generated human OVCAR- 8 cell lines, performed some experiments, and contributed to the discussions. F. Edwards and O. Goundiam generated the PLK4OE OVCAR-8 cell line and contributed to the discussions. D. Gogendeau contributed with part of the SakOE, mad2 analysis, and C. Pennetier generated certain antibodies and helped with fly pushing. R. Basto conceived the project, wrote the manuscript, and supervised the project. A. Goupil, M. Nano, and R. Basto interpreted the data, which was discussed between all authors during the preparation of the manuscript. 
Submitted: 1 August 2019

Revised: 13 December 2019

Accepted: 24 January 2020

\section{References}

Adams, R.R., A.A. Tavares, A. Salzberg, H.J. Bellen, and D.M. Glover. 1998. pavarotti encodes a kinesin-like protein required to organize the central spindle and contractile ring for cytokinesis. Genes Dev. 12:1483-1494. https://doi.org/10.1101/gad.12.10.1483

Basto, R., J. Lau, T. Vinogradova, A. Gardiol, C.G. Woods, A. Khodjakov, and J.W. Raff. 2006. Flies without centrioles. Cell. 125:1375-1386. https://doi .org/10.1016/j.cell.2006.05.025

Basto, R., K. Brunk, T. Vinadogrova, N. Peel, A. Franz, A. Khodjakov, and J.W. Raff. 2008. Centrosome amplification can initiate tumorigenesis in flies. Cell. 133:1032-1042. https://doi.org/10.1016/j.cell.2008.05.039

Bielski, C.M., A. Zehir, A.V. Penson, M.T.A. Donoghue, W. Chatila, J. Armenia, M.T. Chang, A.M. Schram, P. Jonsson, C. Bandlamudi, et al. 2018. Genome doubling shapes the evolution and prognosis of advanced cancers. Nat. Genet. 50:1189-1195. https://doi.org/10.1038/s41588-018-0165-1

Conduit, P.T., A. Wainman, and J.W. Raff. 2015. Centrosome function and assembly in animal cells. Nat. Rev. Mol. Cell Biol. 16:611-624. https://doi .org/10.1038/nrm4062

Desai, A., S. Verma, T.J. Mitchison, and C.E. Walczak. 1999. Kin I kinesins are microtubule-destabilizing enzymes. Cell. 96:69-78. https://doi.org/10 .1016/S0092-8674(00)80960-5

Dewhurst, S.M., N. McGranahan, R.A. Burrell, A.J. Rowan, E. Grönroos, D. Endesfelder, T. Joshi, D. Mouradov, P. Gibbs, R.L. Ward, et al. 2014 Tolerance of whole-genome doubling propagates chromosomal instability and accelerates cancer genome evolution. Cancer Discov. 4:175-185. https://doi.org/10.1158/2159-8290.CD-13-0285

Dogterom, M., and B. Yurke. 1997. Measurement of the force-velocity relation for growing microtubules. Science. 278:856-860. https://doi.org/10 .1126/science.278.5339.856

Duncan, A.W., M.H. Taylor, R.D. Hickey, A.E. Hanlon Newell, M.L. Lenzi, S.B. Olson, M.J. Finegold, and M. Grompe. 2010. The ploidy conveyor of mature hepatocytes as a source of genetic variation. Nature. 467: 707-710. https://doi.org/10.1038/nature09414

Endow, S.A., and D.J. Komma. 1998. Assembly and dynamics of an anastral: astral spindle: the meiosis II spindle of Drosophila oocytes. J. Cell Sci. 111: 2487-2495.

Fink, G., L. Hajdo, K.J. Skowronek, C. Reuther, A.A. Kasprzak, and S. Diez. 2009. The mitotic kinesin-14 Ncd drives directional microtubule-microtubule sliding. Nat. Cell Biol. 11:717-723. https://doi.org/10.1038/ncb1877

Fox, D.T., J.G. Gall, and A.C. Spradling. 2010. Error-prone polyploid mitosis during normal Drosophila development. Genes Dev. 24:2294-2302. https://doi.org/10.1101/gad.1952710

Frawley, L.E., and T.L. Orr-Weaver. 2015. Polyploidy. Curr. Biol. 25: R353-R358. https://doi.org/10.1016/j.cub.2015.03.037

Fujiwara, T., M. Bandi, M. Nitta, E.V. Ivanova, R.T. Bronson, and D. Pellman. 2005. Cytokinesis failure generating tetraploids promotes tumorigenesis in p53-null cells. Nature. 437:1043-1047. https://doi.org/10.1038/ nature 04217

Gallaud, E., R. Caous, A. Pascal, F. Bazile, J.P. Gagné, S. Huet, G.G. Poirier, D. Chrétien, L. Richard-Parpaillon, and R. Giet. 2014. Ensconsin/Map7 promotes microtubule growth and centrosome separation in Drosophila neural stem cells. J. Cell Biol. 204:1111-1121. https://doi.org/10.1083/jcb.201311094

Ganem, N.J., and D. Pellman. 2012. Linking abnormal mitosis to the acquisition of DNA damage. J. Cell Biol. 199:871-881. https://doi.org/10.1083/ jcb.201210040

Ganem, N.J., S.A. Godinho, and D. Pellman. 2009. A mechanism linking extra centrosomes to chromosomal instability. Nature. 460:278-282. https:// doi.org/10.1038/nature08136

Gatti, M., and B.S. Baker. 1989. Genes controlling essential cell-cycle functions in Drosophila melanogaster. Genes Dev. 3:438-453. https://doi.org/ 10.1101/gad.3.4.438

Gemble, S., A. Simon, C. Pennetier, M. Dumont, S. Herve, F. Meitinger, K. Oegema, R. Rodriguez, G. Almouzni, D. Fachinetti, and R. Basto. 2019. Centromere Dysfunction Compromises Mitotic Spindle Pole Integrity. Curr. Biol. 29:3072-3080.e5. https://doi.org/10.1016/j.cub.2019.07.052

Gittes, F., B. Mickey, J. Nettleton, and J. Howard. 1993. Flexural rigidity of microtubules and actin filaments measured from thermal fluctuations in shape. J. Cell Biol. 120:923-934. https://doi.org/10.1083/jcb.120.4.923
Godinho, S.A., R. Picone, M. Burute, R. Dagher, Y. Su, C.T. Leung, K. Polyak, J.S. Brugge, M. Théry, and D. Pellman. 2014. Oncogene-like induction of cellular invasion from centrosome amplification. Nature. 510:167-171. https://doi.org/10.1038/nature13277

Gogendeau, D., K. Siudeja, D. Gambarotto, C. Pennetier, A.J. Bardin, and R. Basto. 2015. Aneuploidy causes premature differentiation of neural and intestinal stem cells. Nat. Commun. 6:8894. https://doi.org/10.1038/ ncomms 9894

Goshima, G., M. Mayer, N. Zhang, N. Stuurman, and R.D. Vale. 2008. Aug min: a protein complex required for centrosome-independent microtubule generation within the spindle. J. Cell Biol. 181:421-429. https://doi .org/10.1083/jcb.200711053

Hayward, D., J. Metz, C. Pellacani, and J.G. Wakefield. 2014. Synergy between multiple microtubule-generating pathways confers robustness to centrosome-driven mitotic spindle formation. Dev. Cell. 28:81-93. https://doi.org/10.1016/j.devcel.2013.12.001

Holland, A.J., D. Fachinetti, Q. Zhu, M. Bauer, I.M. Verma, E.A. Nigg, and D.W Cleveland. 2012. The autoregulated instability of Polo-like kinase 4 limits centrosome duplication to once per cell cycle. Genes Dev. 26: 2684-2689. https://doi.org/10.1101/gad.207027.112

Homem, C.C., and J.A. Knoblich. 2012. Drosophila neuroblasts: a model for stem cell biology. Development. 139:4297-4310. https://doi.org/10.1242/dev.080515

Houchmandzadeh, B., J.F. Marko, D. Chatenay, and A. Libchaber. 1997. Elasticity and structure of eukaryote chromosomes studied by micromanipulation and micropipette aspiration. J. Cell Biol. 139:1-12. https:// doi.org/10.1083/jcb.139.1.1

Howard, J., A.J. Hudspeth, and R.D. Vale. 1989. Movement of microtubules by single kinesin molecules. Nature. 342:154-158. https://doi.org/10.1038/ $342154 \mathrm{a} 0$

Ikeshima-Kataoka, H., J.B. Skeath, Y. Nabeshima, C.Q. Doe, and F. Matsuzaki. 1997. Miranda directs Prospero to a daughter cell during Drosophila asymmetric divisions. Nature. 390:625-629. https://doi.org/10.1038/37641

Kapoor, T.M., T.U. Mayer, M.L. Coughlin, and T.J. Mitchison. 2000. Probing spindle assembly mechanisms with monastrol, a small molecule inhibitor of the mitotic kinesin, Eg5. J. Cell Biol. 150:975-988. https://doi .org/10.1083/jcb.150.5.975

Kirkham, M., T. Müller-Reichert, K. Oegema, S. Grill, and A.A. Hyman. 2003. SAS-4 is a C. elegans centriolar protein that controls centrosome size. Cell. 112:575-587. https://doi.org/10.1016/S0092-8674(03)00117-X

Kwon, M., S.A. Godinho, N.S. Chandhok, N.J. Ganem, A. Azioune, M. Thery, and D. Pellman. 2008. Mechanisms to suppress multipolar divisions in cancer cells with extra centrosomes. Genes Dev. 22:2189-2203. https:// doi.org/10.1101/gad.1700908

Lacroix, B., G. Letort, L. Pitayu, J. Sallé, M. Stefanutti, G. Maton, A.M. Ladouceur, J.C. Canman, P.S. Maddox, A.S. Maddox, et al. 2018. Microtubule Dynamics Scale with Cell Size to Set Spindle Length and Assembly Timing. Dev. Cell. 45:496-511.e6. https://doi.org/10.1016/j devcel.2018.04.022

Letort, G., F. Nedelec, L. Blanchoin, and M. Théry. 2016. Centrosome centering and decentering by microtubule network rearrangement. Mol. Biol. Cell. 27:2833-2843. https://doi.org/10.1091/mbc.e16-06-0395

Letort, G., I. Bennabi, S. Dmitrieff, F. Nedelec, M.H. Verlhac, and M.E. Terret. 2019. A computational model of the early stages of acentriolar meiotic spindle assembly. Mol. Biol. Cell. 30:863-875. https://doi.org/10.1091/ mbc.E18-10-0644

Lucas, E.P., and J.W. Raff. 2007. Maintaining the proper connection between the centrioles and the pericentriolar matrix requires Drosophila centrosomin. J. Cell Biol. 178:725-732. https://doi.org/10.1083/jcb.200704081

Marthiens, V., M. Piel, and R. Basto. 2012. Never tear us apart--the importance of centrosome clustering. J. Cell Sci. 125:3281-3292. https://doi .org/10.1242/jcs.094797

Martinez-Campos, M., R. Basto, J. Baker, M. Kernan, and J.W. Raff. 2004. The Drosophila pericentrin-like protein is essential for cilia/flagella function, but appears to be dispensable for mitosis. J. Cell Biol. 165:673-683. https://doi.org/10.1083/jcb.200402130

Musacchio, A. 2015. The Molecular Biology of Spindle Assembly Checkpoint Signaling Dynamics. Curr. Biol. 25:R1002-R1018. https://doi.org/10 .1016/j.cub.2015.08.051

Musacchio, A., and K.G. Hardwick. 2002. The spindle checkpoint: structural insights into dynamic signalling. Nat. Rev. Mol. Cell Biol. 3:731-741. https://doi.org/10.1038/nrm929

Nano, M., S. Gemble, A. Simon, C. Pennetier, V. Fraisier, V. Marthiens, and R. Basto. 2019. Cell-Cycle Asynchrony Generates DNA Damage at Mitotic Entry in Polyploid Cells. Curr. Biol. 29:3937-3945.e7. https://doi.org/10 .1016/j.cub.2019.09.041 
Nedelec, F., and D. Foethke. 2007. Collective Langevin dynamics of flexible cytoskeletal fibers. New J. Phys. 9:427. https://doi.org/10.1088/1367 $-2630 / 9 / 11 / 427$

Oladipo, A., A. Cowan, and V. Rodionov. 2007. Microtubule motor Ncd induces sliding of microtubules in vivo. Mol. Biol. Cell. 18:3601-3606. https://doi.org/10.1091/mbc.e06-12-1085

Orr-Weaver, T.L. 2015. When bigger is better: the role of polyploidy in organogenesis. Trends Genet. 31:307-315. https://doi.org/10.1016/j.tig.2015 .03 .011

Paim, L.M.G., and G. FitzHarris. 2019. Tetraploidy causes chromosomal instability in acentriolar mouse embryos. Nat. Commun. 10:4834. https:// doi.org/10.1038/s41467-019-12772-8

Prosser, S.L., and L. Pelletier. 2017. Mitotic spindle assembly in animal cells: a fine balancing act. Nat. Rev. Mol. Cell Biol. 18:187-201. https://doi.org/10 $.1038 / \mathrm{nrm} .2016 .162$

Quintyne, N.J., J.E. Reing, D.R. Hoffelder, S.M. Gollin, and W.S. Saunders. 2005. Spindle multipolarity is prevented by centrosomal clustering. Science. 307:127-129. https://doi.org/10.1126/science.1104905

Rhys, A.D., P. Monteiro, C. Smith, M. Vaghela, T. Arnandis, T. Kato, B. Leitinger, E. Sahai, A. McAinsh, G. Charras, and S.A. Godinho. 2018. Loss of E-cadherin provides tolerance to centrosome amplification in epithelial cancer cells. J. Cell Biol. 217:195-209. https://doi.org/10.1083/jcb .201704102

Rupp, B., and F. Nédélec. 2012. Patterns of molecular motors that guide and sort filaments. Lab Chip. 12:4903-4910. https://doi.org/10.1039/c2lc40250e

Santaguida, S., A. Tighe, A.M. D'Alise, S.S. Taylor, and A. Musacchio. 2010. Dissecting the role of MPS1 in chromosome biorientation and the spindle checkpoint through the small molecule inhibitor reversine. J. Cell Biol. 190:73-87. https://doi.org/10.1083/jcb.201001036

Silkworth, W.T., I.K. Nardi, L.M. Scholl, and D. Cimini. 2009. Multipolar spindle pole coalescence is a major source of kinetochore mis-attachment and chromosome mis-segregation in cancer cells. PLoS One. 4:e6564. https:// doi.org/10.1371/journal.pone.0006564

Somma, M.P., B. Fasulo, G. Cenci, E. Cundari, and M. Gatti. 2002. Molecular dissection of cytokinesis by RNA interference in Drosophila cultured cells. Mol. Biol. Cell. 13:2448-2460. https://doi.org/10.1091/mbc.01-12 $-0589$

Storchova, Z., and D. Pellman. 2004. From polyploidy to aneuploidy, genome instability and cancer. Nat. Rev. Mol. Cell Biol. 5:45-54. https://doi.org/ $10.1038 / \mathrm{nrm} 1276$

Straight, A.F., C.M. Field, and T.J. Mitchison. 2005. Anillin binds nonmuscle myosin II and regulates the contractile ring. Mol. Biol. Cell. 16:193-201. https://doi.org/10.1091/mbc.e04-08-0758

Unhavaithaya, Y., and T.L. Orr-Weaver. 2012. Polyploidization of glia in neural development links tissue growth to blood-brain barrier integrity. Genes Dev. 26:31-36. https://doi.org/10.1101/gad.177436.111

Vargas-Hurtado, D., J.B. Brault, T. Piolot, L. Leconte, N. Da Silva, C. Pennetier, A. Baffet, V. Marthiens, and R. Basto. 2019. Differences in Mitotic Spindle Architecture in Mammalian Neural Stem Cells Influence Mitotic Accuracy during Brain Development. Curr. Biol. 29:2993-3005.e9. https://doi.org/10.1016/j.cub.2019.07.061

Watanabe, S., G. Shioi, Y. Furuta, and G. Goshima. 2016. Intra-spindle Microtubule Assembly Regulates Clustering of Microtubule-Organizing Centers during Early Mouse Development. Cell Reports. 15:54-60. https://doi.org/10.1016/j.celrep.2016.02.087

Zack, T.I., S.E. Schumacher, S.L. Carter, A.D. Cherniack, G. Saksena, B. Tabak, M.S. Lawrence, C.Z. Zhsng, J. Wala, C.H. Mermel, et al. 2013. Pancancer patterns of somatic copy number alteration. Nat. Genet. 45: 1134-1140. https://doi.org/10.1038/ng.2760

Zhu, S., S. Lin, C.F. Kao, T. Awasaki, A.S. Chiang, and T. Lee. 2006. Gradients of the Drosophila Chinmo BTB-zinc finger protein govern neuronal temporal identity. Cell. 127:409-422. https://doi.org/10.1016/j.cell.2006.08.045 


\section{Supplemental material}



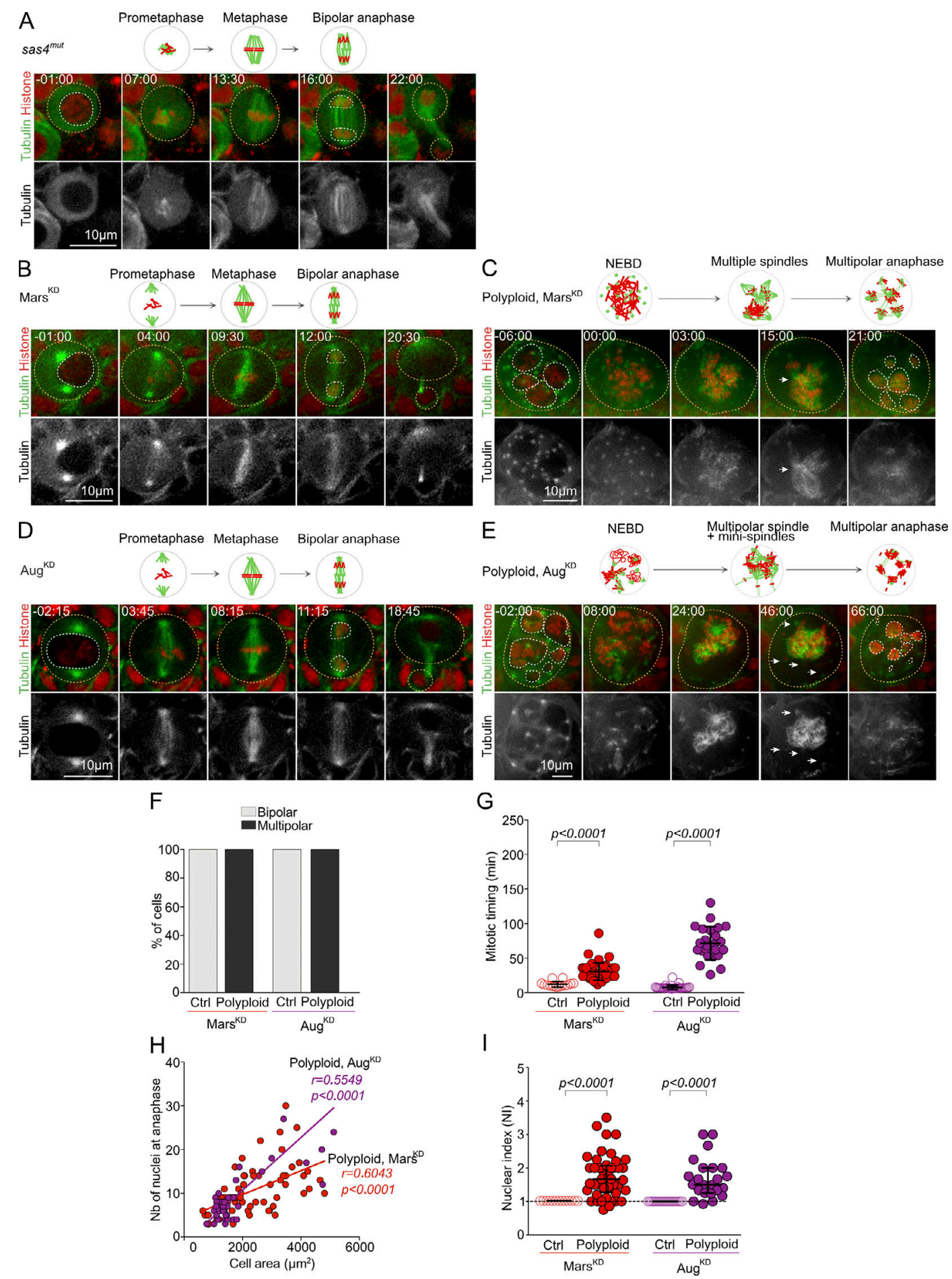

Figure S1. Diploid and polyploid NBs depleted for MT-nucleating pathways undergo bipolar and multipolar divisions. (A-E) Stills of time-lapse videos of mitotic NBs expressing histone-RFP (red) and tubulin-GFP (green and gray in the bottom insets). Orange and white dotted circles surround cells and nuclei, respectively. Time of mitosis is represented in minutes:seconds, and time 00:00 corresponds to NEBD. Schematic representations of mitosis are shown above the stills. Diploid sas4 ${ }^{m u t}(A)$ Diploid, Mars ${ }^{\mathrm{KD}}(\mathrm{B})$, polyploid, Mars ${ }^{\mathrm{KD}}(\mathrm{C})$, diploid, Aug ${ }^{\mathrm{KD}}(\mathrm{D})$, and polyploid, Aug ${ }^{\mathrm{KD}}(\mathrm{E}) \mathrm{NBs}$. (F) Graph bars showing the percentage of cells presenting bipolar and multipolar mitosis in Ctrl, Mars ${ }^{\mathrm{KD}}(n=13 \mathrm{NBs}$ from $1 \mathrm{BL})$, polyploid, Mars ${ }^{\mathrm{KD}}(n=63 \mathrm{NBs}$ from $36 \mathrm{BL}), \mathrm{Ctrl}, \mathrm{Aug}$ KD $(n=30 \mathrm{NBs}$ from $3 \mathrm{BL}$ ), and polyploid, Aug $\mathrm{KD}(n=45 \mathrm{NBs}$ from $15 \mathrm{BL}) \mathrm{NBs}$. (G) Dot plot showing the time spent in mitosis for Ctrl, Mars $\mathrm{KD}(n=13 \mathrm{NBs}$ from $1 \mathrm{BL})$, polyploid, $\operatorname{Mars}^{\mathrm{KD}}(n=63 \mathrm{NBs}$ from $36 \mathrm{BL}), \mathrm{Ctrl}$, Aug ${ }^{\mathrm{KD}}\left(n=30 \mathrm{NBs}\right.$ from $3 \mathrm{BL}$ ) and polyploid, Aug ${ }^{\mathrm{KD}}(n=24 \mathrm{NBs}$ from $10 \mathrm{BL}) \mathrm{NBs}$. (H) XY plot and corresponding linear regression between the number of nuclei generated at anaphase and cell area of polyploid, Mars ${ }^{\mathrm{KD}}(n=63 \mathrm{NBs}$ from $36 \mathrm{BL})$, and polyploid Aug ${ }^{\mathrm{KD}}(n=45 \mathrm{NBs}$ from $15 \mathrm{BL}$ ) NBs. Statistical significance of the correlation was determined by a Spearman $r$ test. $r$ corresponds to the correlation coefficient. (I) Dot plot showing the $\mathrm{NI}$ in Ctrl, Mars ${ }^{\mathrm{KD}}(n=13 \mathrm{NBs}$ from $1 \mathrm{BL})$, polyploid, Mars ${ }^{\mathrm{KD}}(n=44 \mathrm{NBs}$ from 26 brains), Ctrl, Aug $\mathrm{KD}(n=30 \mathrm{NBs}$ from $3 \mathrm{BL})$, and polyploid, Aug $\mathrm{KD}$ ( $n=24$ NBs from 10 brains) NBs. ( $G$ and $\mathbf{I})$ Statistical significance was determined using a Mann-Whitney test. Error bars represent the mean \pm SD $(G)$ and the median \pm interquartile range (I). $\mathrm{p}$ corresponds to the $\mathrm{P}$ value. 
A

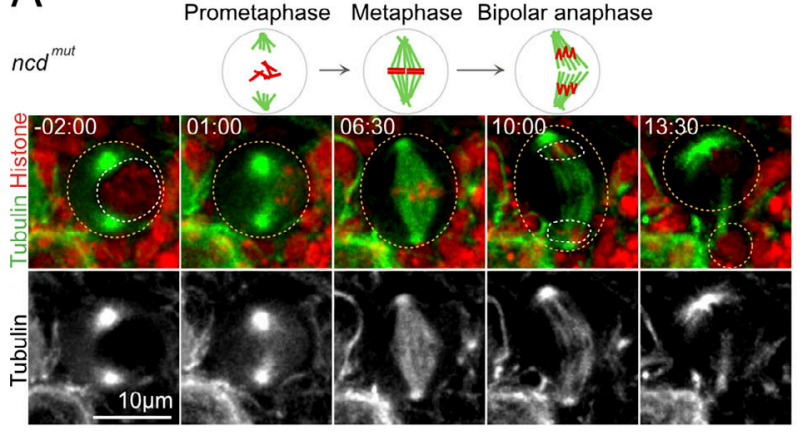

C
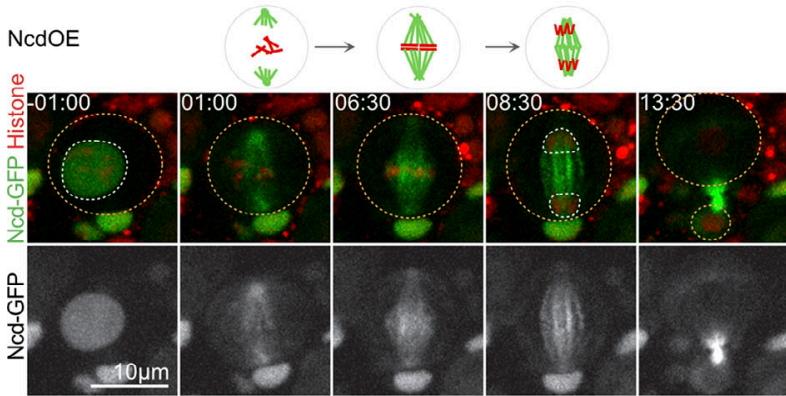

Prometaphase Metaphase Bipolar anaphase

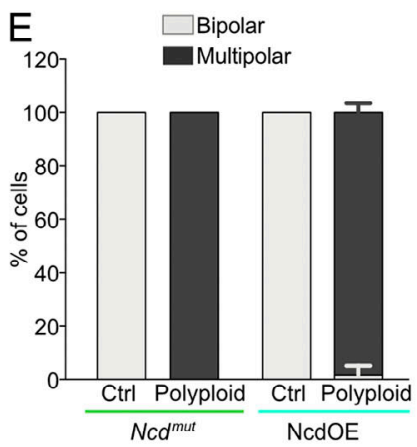

G

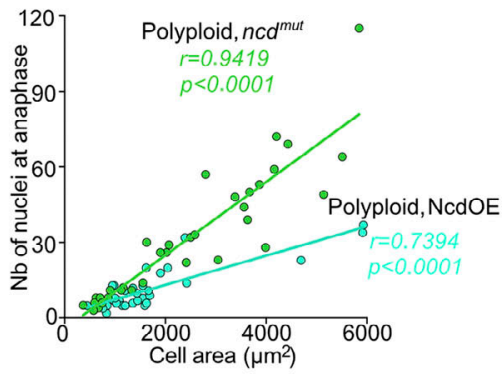

B NEBD Multiple mini-spindles Multipolar anaphase

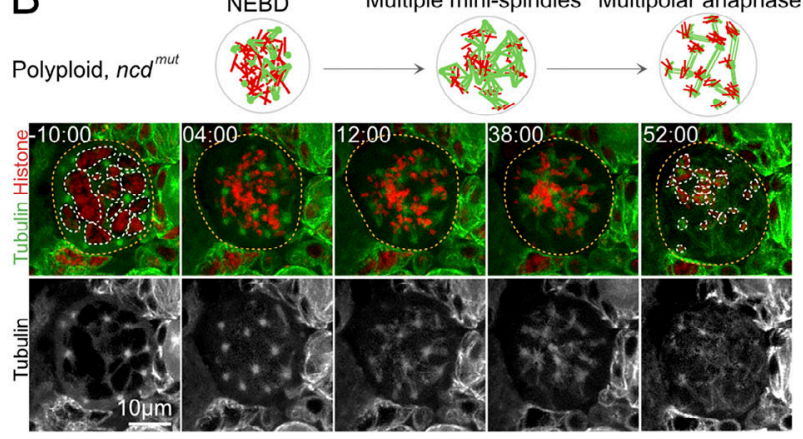

Asynchronous NEBD Multipolar spindle Multipolar anaphase

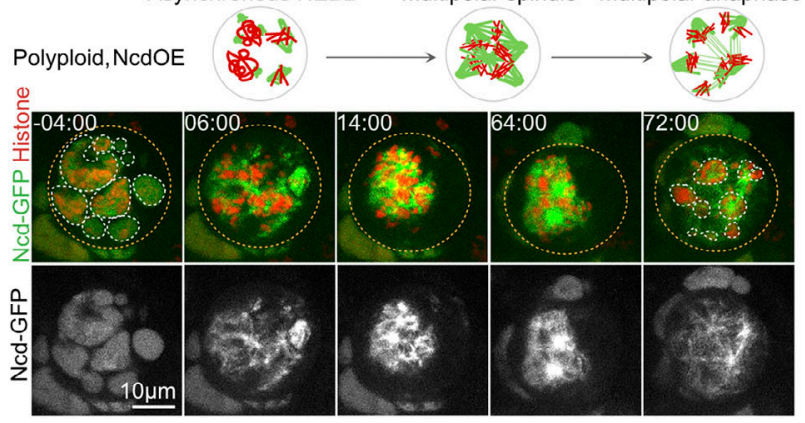

$\mathrm{F}$

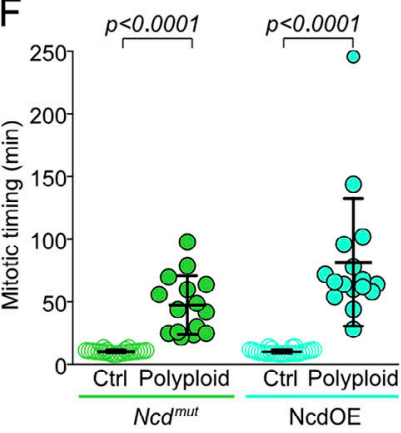

$\mathrm{H}$

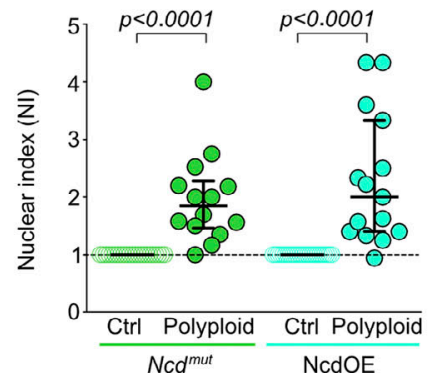

Figure S2. Centrosome clustering takes place in polyploid mitosis, but it is not sufficient to promote bipolar spindle assembly. (A-D) Stills of timelapse videos of mitotic NBs expressing histone-RFP (red) and tubulin-GFP (A and B) or Ncd-GFP (C and D; green). Orange and white dotted circles surround cells and nuclei, respectively. Time of mitosis is represented in minutes:seconds, and time 00:00 corresponds to NEBD. Schematic representations of mitosis are shown above the stills. Ctrl, ncdmut (A), polyploid, $n c d^{m u t}(B), C t r l, N c d O E(C)$, and polyploid, NcdOE (D) NBs. (E) Graph bars showing the percentage of cells undergoing bipolar and multipolar mitosis in Ctrl, $n c d^{\text {mut }}\left(n=23 \mathrm{NBs}\right.$ from $3 \mathrm{BL}$ ), polyploid, $n$ nd $^{\text {mut }}(n=34 \mathrm{NBs}$ from $20 \mathrm{BL}), \mathrm{Ctrl}, \mathrm{NcdOE}(n=25 \mathrm{from} 2 \mathrm{BL})$, and polyploid, $\operatorname{NcdOE}(n=35$ from $6 \mathrm{BL}) \mathrm{NBs}$. (F) Dot plot showing the time spent in mitosis for Ctrl, $n c d^{m u t}(n=24 \mathrm{NBs}$ from $3 \mathrm{BL})$, polyploid, $n c d^{m u t}(n=15 \mathrm{NBs}$ from $12 \mathrm{BL}$ ), Ctrl, NcdOE ( $n=25 \mathrm{NBs}$ from $2 \mathrm{BL}$ ), and polyploid, NcdOE ( $n=16 \mathrm{NBs}$ from $5 \mathrm{BL}$ ) NBs. (G) XY plot and corresponding linear regression between the number of nuclei generated at anaphase and cell area for polyploid, $n c d^{m u t}(n=34 \mathrm{NBs}$ from $20 \mathrm{BL}$ ) and polyploid, NcdOE $(n=35 \mathrm{NBs}$ from $6 \mathrm{BL}) \mathrm{NBs}$. Statistical significance of the correlation was determined by a Spearman $r$ test. $r$ corresponds to the correlation coefficient. (H) Dot plot showing the NI for $\mathrm{Ctrl}$, $n d^{\text {mut }}\left(n=24 \mathrm{NBs}\right.$ from $3 \mathrm{BL}$ ), polyploid, $n c d^{\text {mut }}(n=14 \mathrm{NBs}$ from $11 \mathrm{BL}$ ), Ctrl, NcdOE ( $n=25 \mathrm{NBs}$ from $2 \mathrm{BL}$ ), and polyploid NcdOE ( $n=15 \mathrm{NBs}$ from $4 \mathrm{BL})$ NBs. ( $F$ and $H$ ) Statistical significance was determined using a Mann-Whitney test. Error bars represent the mean \pm SD $(F)$ and the median \pm interquartile range $(H)$. $p$ corresponds to the $P$ value. 
A

Bipolar without chromosome barrier
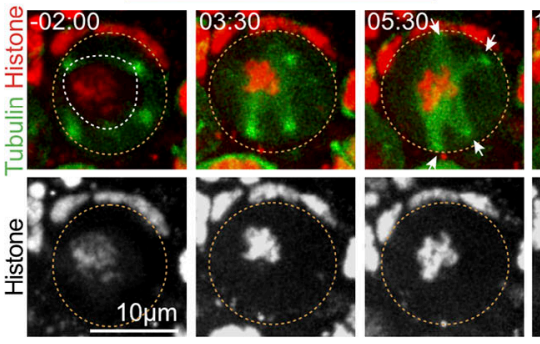

C

Pseudo-bipolar with chromosome barrier
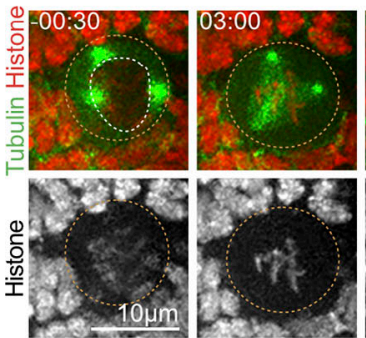

E

B

Bipolar with chromosome barrier
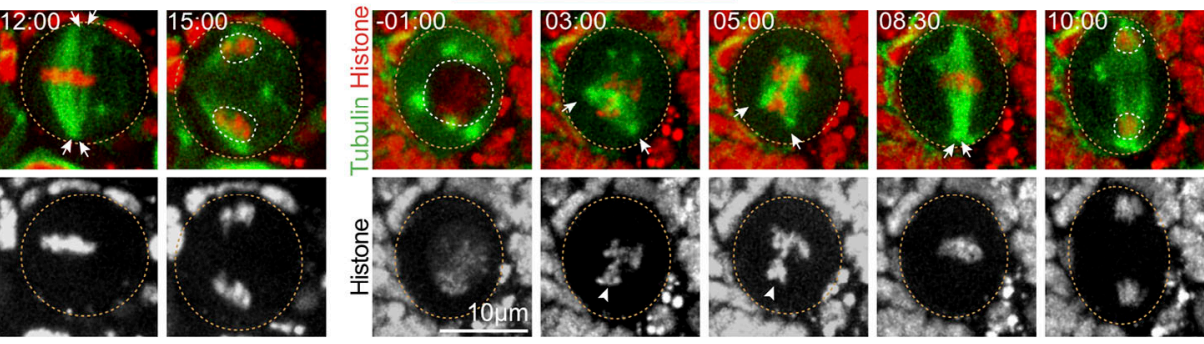

1)

D

Multipolar with chromosome barrier
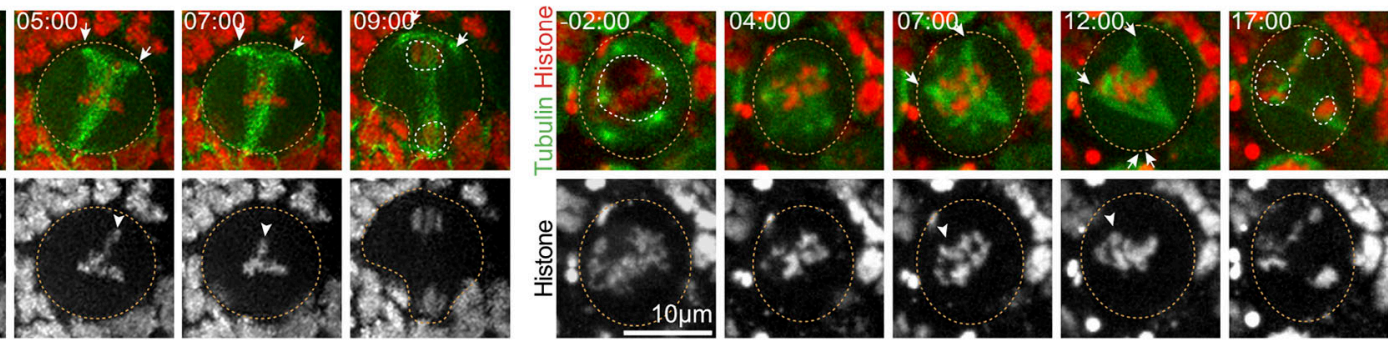

chromosome(s) barrier?
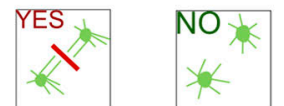

$n=9(47 \%)$

$n=10(53 \%)$

SakOE, $\operatorname{mad} 2^{m}$
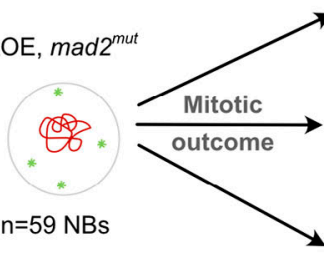

Bipolar

$n=19(32 \%)$

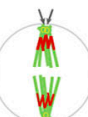

Pseudo-bipola

$n=18(31 \%)$

$n=59$ NBs

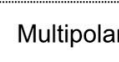

$n=22(37 \%)$

N

$n=19(86 \%) \quad n=3(14 \%)$

Figure S3. Chromosomes as physical barrier can perturb centrosome clustering in diploid cells with centrosome amplification. (A-D) Stills of timelapse videos of mitotic SakOE, mad2mut NBs expressing tubulin-GFP (green) and histone-RFP (red and gray in the bottom insets). Orange and white dotted circles surround cells and nuclei, respectively. Time of mitosis is indicated in minutes:seconds, and time 00:00 corresponds to NEBD. (A) SakOE, mad2 ${ }^{\text {mut }}$ NB with several MT-nucleating sites builds up a bipolar spindle as centrosomes (arrows) are not blocked by a DNA barrier. (B) SakOE, mad2 ${ }^{\text {mut }}$ NB presents several MT-nucleating sites and assembles a tripolar spindle containing DNA (arrowhead) between two poles (arrows). As mitosis proceeds, the DNA is pushed toward the metaphase plate, and the spindle becomes bipolar. (C) SakOE, mad2 ${ }^{\text {mut }}$ NB with several MT-nucleating sites assembles first a tripolar spindle. A DNA mass acts as a barrier blocking the clustering of two poles (arrows). At anaphase onset, a pseudo-bipolar spindle, resulting from partial centrosome clustering, generates two nuclei. (D) SakOE, mad2 ${ }^{\text {mut }}$ NB with several MT-nucleating sites displays multipolar division, as unresolved DNA barrier inhibits pole coalescence. (E) Table showing the number and the percentage of cells in each indicated category in SakOE, mad2 ${ }^{\text {mut }} \mathrm{NBs}(n=59 \mathrm{NBs})$. 
A

Ctrl OVCAR-8 cells (DOX-)

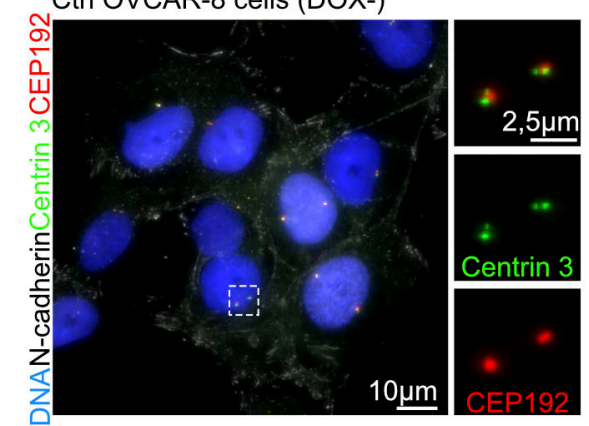

C

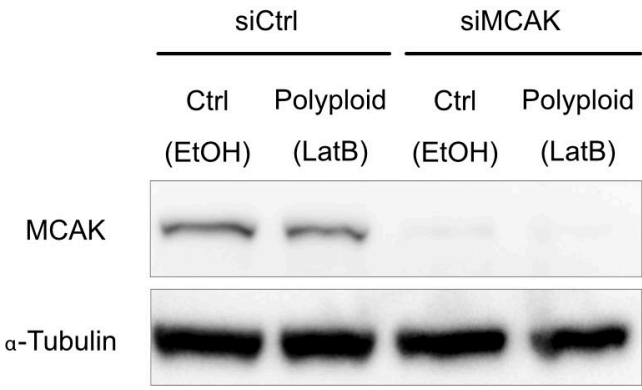

PLK4OE OVCAR- 8 cells (DOX+)

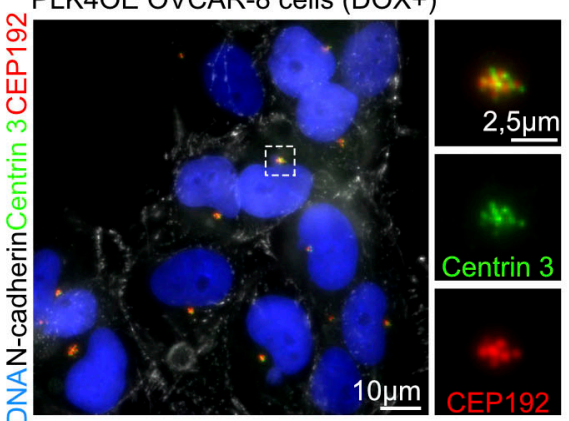

D

Polyploid siCtrl Microtubules

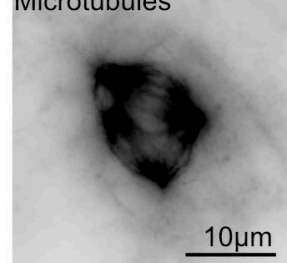

Polyploid siMCAK Microtubules

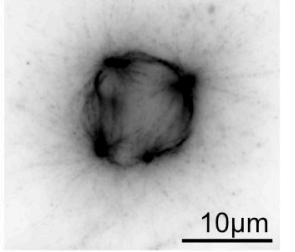

B $\square>4$ centrioles

$\square \leq 4$ centrioles

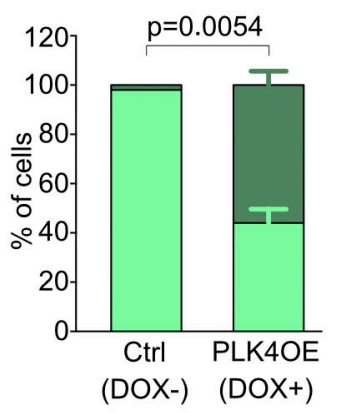

$\left.E^{25}\right] \quad p<0.0001$

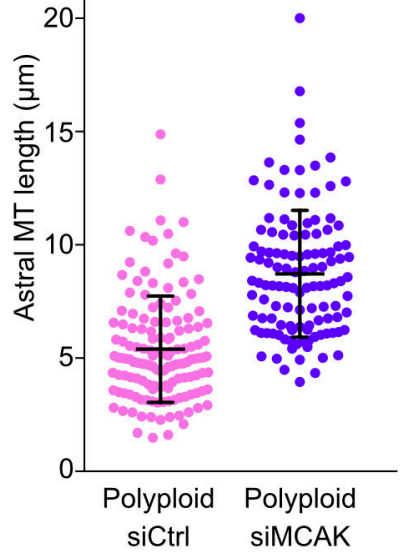

$\mathrm{F}$

Polyploid OVCAR-8 cell with stabilized MTs (siMCAK) - Multipolar
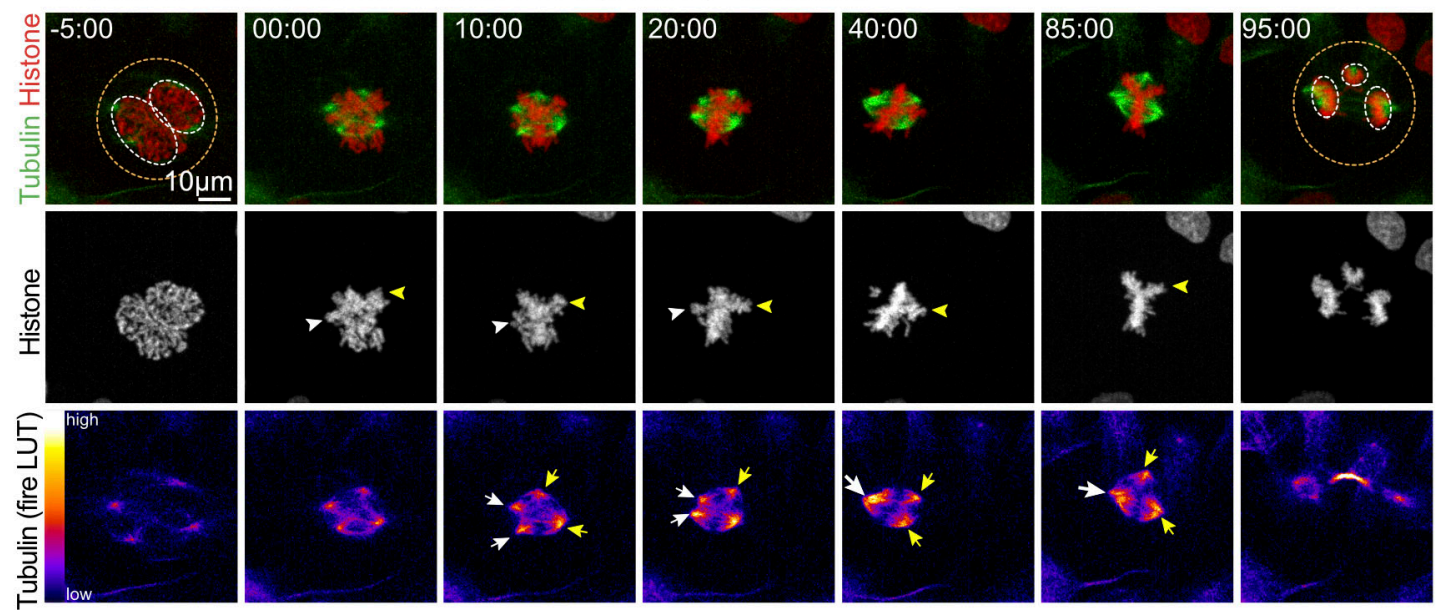

Figure S4. PLK4OE and MCAK depletion, respectively, induce centrosome amplification and increased astral MT length. (A) Pictures of Ctrl (DOX-) and PLK4OE (DOX+) OVCAR- 8 cells labeled with antibodies against CEP192 and Centrin 3 to label the centrosomes and against the membrane marker N-cadherin. (B) Graph bars showing the percentage of Ctrl (DOX-; $n=488$ cells from two independent experiments) and PLK4OE (DOX +; $n=410$ cells from two independent experiments) OVCAR-8 cells presenting four or fewer or more than four centrioles. Statistical significance was determined using a multiple $t$ test. (C) Western blot of siCtrl and siMCAK, OVCAR-8 cells. (D) Pictures of siCtrl polyploid and siMCAK polyploid OVCAR-8 cells labeled with antibodies against a-tubulin. (E) Dot plot showing the length of astral MTs in siCtrl, polyploid ( $n=140$ MTs from 11 cells) and siMCAK, polyploid ( $n=118$ MTs from eight cells) OVCAR-8 cells. Statistical significance was determined using a Mann-Whitney test. (F) Stills of time-lapse video of mitotic polyploid OVCAR-8 cell with stabilized MTs (siMCAK) expressing histone 2B-GFP (red and gray in the middle insets) and incubated with SiR-tubulin (green and fire LUT in the bottom insets). Orange and white dotted circles surround cells and nuclei, respectively. Time of mitosis is represented in minutes:seconds, and time 00:00 corresponds to the NEBD. Error bars represent the mean \pm SD. $p$ corresponds to the $P$ value. 
Video 1. Mitosis in diploid and small and large polyploid NBs. NBs expressing tubulin-GFP (green) and histone-RFP (red). Time of mitosis is indicated in minutes:seconds and hours:minutes:seconds for Ctrl and PavKD NBs, respectively. Frame rate speed of six frames per second. Time 00:00 and 00:00:00 correspond to NEBD. Still images from this video shown in Fig. 1.

Video 2. Mitosis in diploid, sas4 ${ }^{\text {mut }}$ and small and large polyploid, sas4 ${ }^{\text {mut }}$ NBs. NBs expressing tubulin-GFP (green) and histone-RFP (red). Time of mitosis is indicated in minutes:seconds and hours:minutes:seconds for Ctrl and PavKD NBs, respectively. Frame rate speed of six frames per second. Time 00: 00 and 00:00:00 correspond to NEBD. Still images from this video shown in Fig. 3 and Fig. S1.

Video 3. Mitosis in diploid, Mars ${ }^{\mathrm{KD}}$, diploid, Aug ${ }^{\mathrm{KD}}$, diploid, ncd $^{\text {mut }}$, and diploid, NcdOE NBs. NBs expressing histone-RFP (red) and tubulin-GFP (green) or Ncd-GFP (green). Time of mitosis is indicated in minutes:seconds, and time 00:00 corresponds to NEBD. Frame rate speed of six frames per second. Still images from this video shown in Figs. S1 and 2.

Video 4. Mitosis in polyploid, Mars ${ }^{\mathrm{KD}}$, polyploid, Aug ${ }^{\mathrm{KD}}$, polyploid, ncd $^{\text {mut }}$, and polyploid, NcdOE NBs. NBs expressing histone-RFP (red) and yubulinGFP (green) or Ncd-GFP (green). Time of mitosis is indicated in hours:minutes:seconds, and time 00:00:00 corresponds to NEBD. Frame rate speed of six frames per second. Still images from this video shown in Figs. S1 and 2.

Video 5. Centrosome clustering in polyploid NBs. NBs expressing Spd2-GFP (green) and histone-RFP (red). Time of mitosis is indicated in hours:minutes:seconds. Time 00:00:00 corresponds to NEBD. Frame rate speed of six frames per second. Still images from this video are shown in Fig. 4.

Video 6. Computer simulations of spindle assembly according to centrosome number, DNA content, and DNA shape. MTs are represented as green lines, centrosomes as green circles, and DNA as red dots or red plates. Computer simulations of spindle behavior run with low and high number of centrosomes, DNA beads, and different DNA shapes. Frame rate speed of six frames per second. Still images from this video shown in Fig. 4.

Video 7. Mitosis in Ctrl, PLK4OE and polyploid OVCAR-8 cells. Mitotic cells expressing histone 2B-GFP (red) and incubated with SiR-tubulin (green). Time of mitosis is indicated in hours:minutes:seconds, and time 00:00:00 corresponds to NEBD. Frame rate speed of six frames per second. Still images from this video shown in Figs. 5 and 6.

Video 8. Laser ablation in polyploid OVCAR-8 cells. Mitotic cells expressing histone 2B-GFP (red) and incubated with SiR-tubulin (green), presenting or failing spindle pole coalescence after laser ablation. Time of mitosis is indicated in hours:minutes:seconds, and time 00:00:00 corresponds to time of laser ablation. Yellow dotted circles represent laser ablation area. Frame rate speed of six frames per second. Still images from this video shown in Fig. 7.

Video 9. Mitosis in mononucleated polyploid cells. Computer simulations of spindle assembly in conditions of one or multiple DNA bead clusters and mitosis of mononucleated polyploid OVCAR-8 cells expressing histone 2B-GFP (red) and incubated with SiR-tubulin (green). Time of mitosis is indicated in hours:minutes:seconds, and time 00:00:00 corresponds to NEBD. Frame rate speed of six frames per second. Still images from this video shown in Fig. 8.

Video 10. Mitosis in polyploid cells with increased MT stability. Computer simulations of spindle assembly in conditions of Ctrl and increased MT stability and mitosis of polyploid OVCAR-8 cells expressing histone 2B-GFP (red) and incubated with SiR-tubulin (green) with siCtrl or siMCAK. Time of mitosis is indicated in hours:minutes:seconds, and time 00:00:00 corresponds to NEBD. Frame rate speed of six frames per second. Still images from this video shown in Fig. 9. 
Provided online are three supplemental tables in Excel format. Table S1 shows the different parameters of mitotic NBs in diploid and polyploid conditions characterized in this study. The mitotic timing corresponds to the time elapsed from NEBD to anaphase onset in minutes. The $\mathrm{NI}$ is calculated as the ratio between the number of nuclei at anaphase over the number of nuclei at mitotic entry relative to the number of daughter cells. The slope of the linear regression between the number of nuclei generated at anaphase and polyploid cell area is determined using the equation of the linear regression line. Values correspond to the mean \pm SD or percentage, and the statistical significances are related to diploid or polyploid controls. Statistical significances have been determined by Mann-Whitney tests for mitotic timing and NI and by linear regression for the slope lines. ND, not determined; ns, not significant; $\mathrm{p}, \mathrm{P}$ value. Table S2 lists computer simulation parameters. Table S3 lists materials. 